\title{
A SCOPING REVIEW OF THE LITERATURE ON THE RELATIONSHIP BETWEEN SOCIAL AND STRUCTURAL DETERMINANTS \\ OF HEALTH AND NEUROSURGICAL OUTCOMES
}

\author{
A Thesis \\ Submitted to \\ the Temple University Graduate Board
}

In Partial Fulfillment

of the Requirements for the Degree

MASTER OF ARTS

by

Gregory Glauser

Diploma Date: May 2021

Whotney V. Abey
Thesis Approvals:

Whitney Cabey, MD, MSHP, MA, Thesis Advisor, Center for Bioethics, Urban Health, and Policy 


\begin{abstract}
This thesis discloses findings from a scoping review of Social and Structural Determinant of Health (SSDOH)-related academic and grey literature from neurosurgery and fields with neurosurgical overlap. The purpose of this thesis is to identify which social determinants have been assessed, for which surgical procedures they were evaluated, and what disparities were found. To identify studies to include or consider for this systematic review, the review team worked with a medical librarian to develop detailed search strategies for each database. Studies were screened by title and abstract independently by two reviewers. Disagreements between reviewers were resolved by a third reviewer, blinded to the decisions of the primary reviewers.
\end{abstract}

The scoping review of the SSDOH in neurosurgical outcomes identified 99 studies from the year 1990 to 2020 . Identified studies were targeted predominantly toward spine surgery patients, evenly distributed in analyses of gender, race and economic stability. The relatively low volume of neurosurgical papers focused on the SSDOH and emphasis on one subspecialty demonstrates the need for an expanded interest in the SSDOH in neurosurgery. 
For Ariana, Araceli, Mom and Dad for being the most supportive family.

For my grandfather, Kurt, who exemplified what it meant to give back. 


\section{TABLE OF CONTENTS}

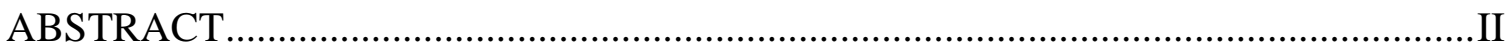

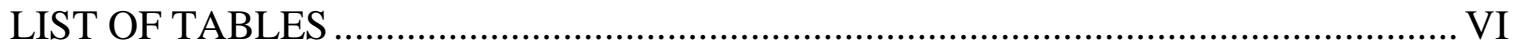

LIST OF FIGURES .................................................................................. VII

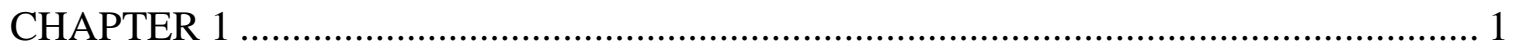

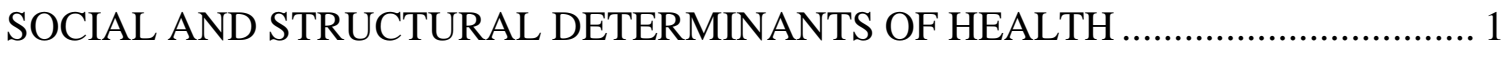

Current State: SSDOH in Medicine ............................................................. 3

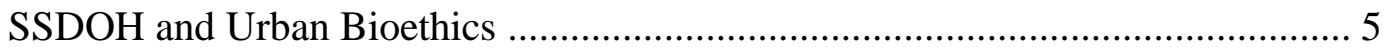

SSDOH and Urban Bioethics for Neurosurgeons ................................ 6

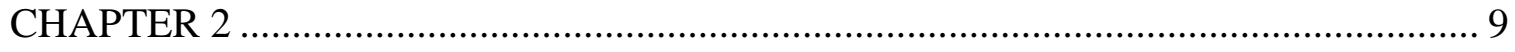

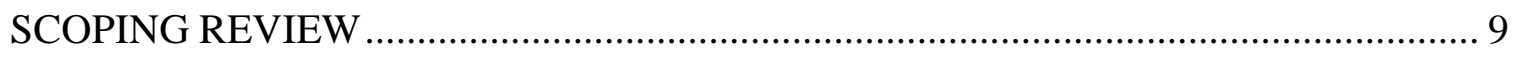

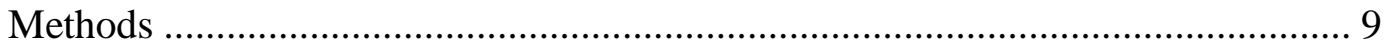

Research Questions ............................................................... 10

Eligibility Criteria .................................................................. 10

Participants ................................................................. 10

Concept ..................................................................... 10

Context ........................................................................ 11

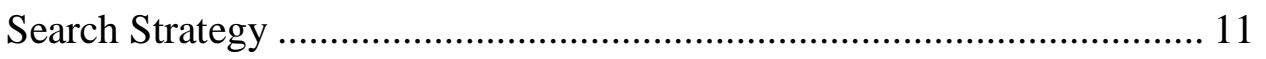

Citation Management and Study Selection ....................................... 12

Data Extraction, Collation and Summary ........................................ 12

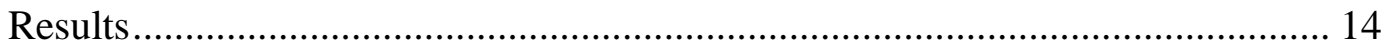

Descriptive Numerical Summary ................................................... 14 
Characteristics of Included Studies................................................... 15

Studied Social Determinants .......................................................... 19

Education ............................................................................ 19

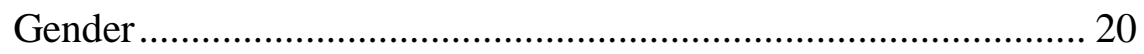

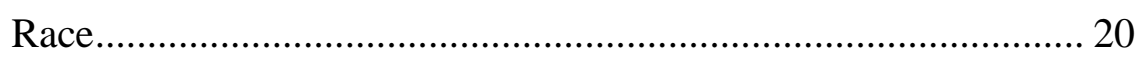

Economic Stability .......................................................... 21

Economic Stability: Insurance Status .................................... 21

Economic Stability: Employment Status ............................... 22

Economic Stability: Household Income ................................ 23

Structural Determinants .............................................................. 23

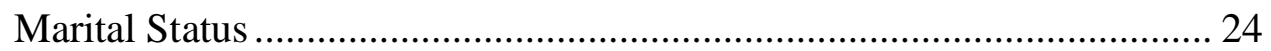

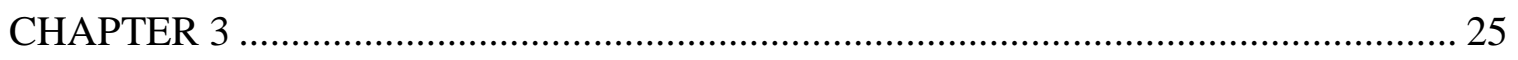

\section{STRUCTURAL AND SOCIAL DETERMINANTS OF HEALTH IN}

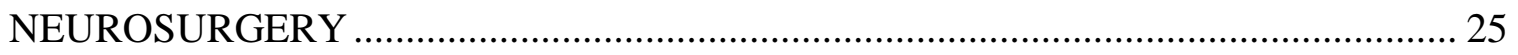

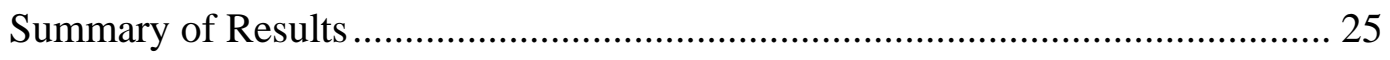

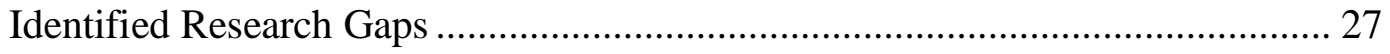

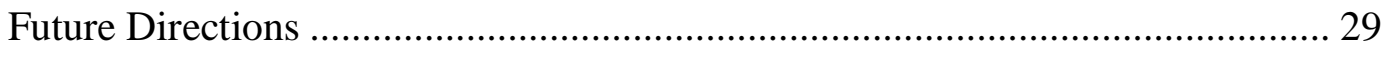

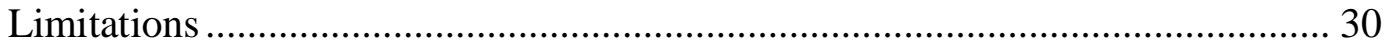

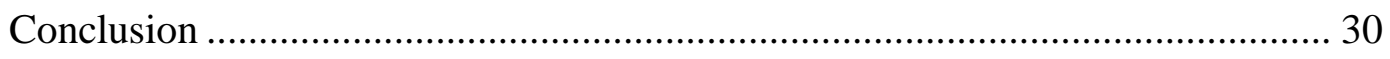

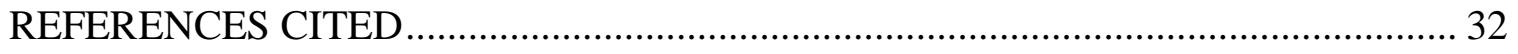

APPENDIX: FULL SEARCH PROTOCOL ............................................................ 38 


\section{LIST OF TABLES}

Table Page

Table 1. Characteristics of Included Studies............................. 17-18

Table 2. Outcomes Assessed in Included Studies............................ 19

Table 3. Studied SSDOH by Neurosurgical Subspecialty..................... 27 


\section{LIST OF FIGURES}

$\begin{array}{lll}\text { Figure Page } & \text { Pas }\end{array}$

Figure 1. PRISMA Flowchart of the

Study Selection Process.

Figure 2. Treemap Showing Proportional Representation of the Number of Completed Studies Evaluating the SSDOH and Neurosurgical Outcomes, Grouped

by Social or Stuctural Determinant of Health.

Figure 3. Treemap Showing Proportional Representation of the Number of Completed Studies Evaluating the SSDOH and Neurosurgical Outcomes, Grouped by Neurosurgical Subspecialty

Figure 4. Line Graph Demonstrating the Trend of Publications Assessing the SSDOH and Neurosurgical Outcomes

Figure 5. Bar Graph Showing the Number of Studies Analyzing the SSDOH in Each Neurosurgical Subspecialty, Based on Individual Social or Structural Determinant.

Figure 6. Bar Graph Showing Correlation of Insurance Status, Employment Status, and Household Income with Outcomes by Neurosurgical Subspecialty 


\section{CHAPTER 1}

\section{SOCIAL AND STRUCTURAL DETERMINANTS OF HEALTH}

The issue of healthcare disparities is largely driven by the social and structural determinants of health (SSDOH), which refer to the complex factors outside of the healthcare setting that impact a patient's health risks and outcomes. In recent years, the SSDOH have gained increasing prominence as a fundamental concept to public health and have gradually carried over in to various medical and surgical specialties. SSDOH are defined by The World Health Organization (WHO) as "The conditions in which people are born, grow, live, work and age" (World Health Assembly, 62, 2009). SSDOH include, but are not limited to, gender, race and economic stability (i.e., household income, insurance status, etc.) (Catalyst, 2017). The SSDOH have been extensively linked to health metrics. One such example is data from the Virginia Commonwealth University Center on Society and Health, showing a 20 year difference in life expectancy between two Philadelphia zip codes (19132 and 19106), which are located a mere 4 miles away from each other (Mapping life expectancy: Philadelphia, 2016).

Though often individually incapable of addressing the aspects of health determinants that are far upstream of delivery, healthcare providers are tasked with managing and attempting to mitigate the consequences. Therefore, it is crucial for providers to have a robust understanding of the impact the SSDOH have on the care they deliver to their patients. Each area of the healthcare experience can contribute to disparities, i.e.: provider of care, access to care and outcome of care subsequent to attainment of access (Smedley, Brian D., Stith, Adrienne Y., Nelson, Alan R., 2003; West et al., 2018). For surgical providers, who typically attain patients through referral, the 
goal of mitigating disparities is most easily accomplished through ensuring post-operative outcomes are equivalent for all patients (Torain et al., 2015). Though not the only avenue for surgeons to act upon disparities, identification of at-risk patients pre-operatively offers surgeons a method for engaging in socially cognizant care. In an effort to inform surgical providers regarding the existent disparities and how to provide equitable care, there has been an expansion in literature evaluating how the SSDOH impact surgical outcomes (Torain et al., 2015).

Beyond the efforts of individual providers, hospitals have clear incentives to monitor and address community needs, particularly in the time since the enactment of the Affordable Care Act. Accounting for the SSDOH may help accountable care organizations reduce costs and meet value-based-care goals, such as mitigating risk of readmission or medication nonadherence (Maani \& Galea, 2020). In this context, delivering value-based-care has become increasingly relevant for providers in the surgical subspecialties.

With the expanding focus, financial incentives, awareness and literature revolving around the SSDOH in the surgical literature, this thesis evaluates the status of knowledge of the SSDOH in one surgical subspecialty - neurosurgery. Interest in the SSDOH in neurosurgery has evolved, as providers are increasingly using data to inform their practice, particularly with respect to identifying factors which may contribute to patient outcomes. 


\section{Current State: SSDOH in Medicine}

Focus on the SSDOH in medicine has intensified in the latter half of the 2010's. This is likely in response to both the value-based-care incentives of the Affordable Care Act and a growing awareness of the SSDOH. At the end of the 2010's, three American medical societies releasing formal position statements on their respective specialty's role in addressing health disparities (Advancing health equity by addressing the social determinants of health in family medicine (position paper).; Byhoff et al., 2020; Daniel, Bornstein, \& Kane, 2018a). These groups, the American College of Physicians, the Society of General Internal Medicine and the American Academy of Family Physicians (AAFP), are societies of primary care providers. This reflects a widely held notion that health disparities are under the purview of primary care, as surgeons are often receiving patients on a referral basis and are tasked with treating a specific pathology. The AAFP alludes to this in its position statement by noting that family physicians provide highquality healthcare for underserved populations more so than other medical specialties (Advancing health equity by addressing the social determinants of health in family medicine (position paper).).

However, the distinct action items posited by the AAFP position statement are steps that can readily apply to all healthcare providers. These include advocating for public policies that address SSDOH, knowing which organizations are working to improve health equity in your community and understanding what health inequities exist within your community and helping raise the prominence of these issues(Advancing health equity by addressing the social determinants of health in family medicine (position 
paper).) While these steps may require varying degrees of activism and community engagement, the remaining steps proposed by the AAFP can be a seamless part of any physician's practice. These steps include creating a practice culture that values health equity by addressing implicit bias in your practice, using cultural proficiency and health literacy standards, knowing how patients are affected by SSDOH and helping address their needs to improve their health(Advancing health equity by addressing the social determinants of health in family medicine (position paper).). For these aims to be met, it is vitally important that the conversation around the SSDOH continues to evolve across medical and surgical specialties.

The position statement from the Society of General Internal Medicine similarly presents multiple action items which can be integrated across specialties. Of note, the position statement emphasizes the need for working in interprofessional care teams to ensure whole-person care is delivered for all patients (Byhoff et al., 2020). The authors posit that in order to adequately identify and address complex medical and social needs in a clinical setting, multidisciplinary care teams are essential. Necessary stakeholders include social workers, community health workers, pharmacists, mental health, nurses and front-line/administrative staff. With this team-based expertise, providers are able to deliver support tailored to the unique needs of each and every patient, accounting for factors impacted by the SSDOH (Byhoff et al., 2020). The American College of Physicians position statement echoes this sentiment, offering support for increased interprofessional communication and collaborative models that encourage a team-based approach to treating patients at risk to be negatively affected by the SSDOH (Daniel, Bornstein, \& Kane, 2018b). 
Each of these position statements relays, to varying degrees, a need for increased use of healthcare data to drive studies evaluating how the SSDOH impact patients. Although these societies are composed of primary care providers, each of the aforementioned approaches for providing equitable care are applicable and can be readily integrated to other specialties. With regard to societies of surgical providers, the American College of Surgeons put forth a short statement in 2013 acknowledging that the SSDOH affect access to surgical care (Statement on optimal access, 2013). Otherwise, no societies of surgery have put forth statements discussing how access to surgical care or outcomes of surgical care are impacted by the SSDOH.

\section{SSDOH and Urban Bioethics}

Interest in the SSDOH for physicians in all specialties aligns with a number of important bioethical principles. SSDOH are known to contribute to health inequities, which are defined as differences or disparities in outcomes that are systematic, unavoidable, and unjust (Advancing health equity by addressing the social determinants of health in family medicine (position paper).). Doctors are faced daily with the consequences of health disparities, defined as differences in health that are closely linked with social or economic disadvantage (Advancing health equity by addressing the social determinants of health in family medicine (position paper).). Disparities in healthcare impact groups of people who have systematically experienced greater economic or social barriers to health (Byhoff et al., 2020). Therefore, in order for physicians to best help 
their patients, regardless of medical specialty, it is necessary for all providers to understand how the SSDOH may impact their patient.

Providing ethically focused care requires that physicians aim to generate health equity, which means that everyone has a fair and just opportunity to be as healthy as possible (Advancing health equity by addressing the social determinants of health in family medicine (position paper).). Achieving health equity requires removing obstacles to health such as poverty, discrimination, and their consequences. In order to help their patients to remove obstacles, physicians must come to understand what obstacles their patients face. Understanding the role of SSDOH and working towards improving upon health inequity aligns with the principles of medical ethics and urban bioethics, which serve as guiding principles for all healthcare providers.

\section{SSDOH and Urban Bioethics for Neurosurgeons}

For neurosurgeons, understanding the SSDOH follows the principles of respect for autonomy and agency. With respect to autonomy in healthcare decision making, it is implied that the patient is capable of acting intentionally, with full understanding, and without controlling influences that would diminish a voluntary and free act (McCormick, 2018). In urban bioethics, agency takes the principle of autonomy a step further referring to ones ability to see a complete range of options in a given situation, dependent on the individuals life experiences (Blacksher \& Lovasi, 2012; Blustein \& Fleischman, 2004). Neurosurgical procedures are intricate and often urgent, requiring dense explanation and 
quick decision making for providers and patients alike. Physician understanding of issues such as cultural mistrust of the healthcare system may help neurosurgeons in having well received and thorough informed consent conversations that can improve patient trust. Neurosurgeons who can appreciate patient agency by accounting for the SSDOH will successfully position their patients to understand and follow the guidance they deliver.

Being versed in the SSDOH aligns with the principles of beneficence and solidarity for neurosurgeons. Beneficence means that healthcare providers have a duty to be of benefit to the patient, as well as to take positive steps to prevent and to remove harm from the patient (McCormick, 2018). In urban bioethics, solidarity binds multiple stakeholders together, taking in to consideration that the external forces that led one stakeholder to be advantaged also led the other party to be disadvantaged (Blacksher \& Lovasi, 2012; Blustein \& Fleischman, 2004). This principle ties the stakeholders together in a mutually beneficial way, where each party exists for the other. An example of this is if data demonstrates to a spine surgeon that low-income patients undergoing a lumbar fusion are at an increased risk of hospital readmission. This understanding can empower the surgeon to work proactively with social work and other stakeholders in the care coordination team to ensure that the patient has adequate support in place prior to discharge. Coordinated efforts such as this benefit both the patient and provider, as they avoid a negative post-operative outcome.

Knowledge of the SSDOH for neurosurgeons follows the principle of nonmaleficence by helping providers avoid unreasonable risk of harm. This principle entails that harm or injury is not created for the patient, whether by acts of commission of 
omission (McCormick, 2018). Further, understanding the SSDOH enables neurosurgeons to follow the principle of justice, which implies fair treatment for all (McCormick, 2018). Urban bioethics expands this principle to social justice, which requires consideration of contextual and structural inequities when allocating resources (Blacksher \& Lovasi, 2012; Blustein \& Fleischman, 2004). The principle of social justice posits that in order to deliver fair treatment, it is necessary to acknowledge that treatment may differ based on factors that impact the overall need for resources.

In order to assure that all of their patients are receiving equitable treatment, neurosurgeons need to use a knowledge of the SSDOH to understand what is required by them to avoid an unreasonable risk of harm and deliver fair treatment. For example, if a pediatric neurosurgeon has data which demonstrates that patients who live in a particular zip code have unfavorable outcomes following brain tumor resection, it is incumbent on the care team to ensure that the identified at-risk patient has support in place to receive fair treatment. The care team can identify what inequities exist for the patient and allocate additional resources as necessary. Therefore, data on the SSDOH can be utilized by the neurosurgeon and care coordinators to help deliver equitable care, aligning with both the urban bioethical principles of nonmaleficence and social justice. 


\section{CHAPTER 2}

\section{SCOPING REVIEW}

Neurosurgical procedures are complex and costly, so it behooves providers to understand all of the factors which may influence their patient's overall health and surgical outcome. While interest in this area is growing, there have been no reviews of the SSDOH in neurosurgery. To date, there has not been a comprehensive evaluation of which social determinants have been assessed, for which procedures they were evaluated, and what disparities were found. This chapter reports on findings from a scoping review of SSDOH-related academic and grey literature from neurosurgery and fields with neurosurgical overlap (endovascular neurointervention, spinal deformity, pituitary surgery, etc.).

\section{Methods}

The findings of this scoping review are reported in accordance with the Preferred Reporting Items for Systematic Reviews and Meta-Analysis (PRISMA) Extension for Scoping Reviews (PRISMA-ScR) Checklist (Tricco et al., 2018). Further, the review is guided by the 2005 methodological framework for conducting scoping reviews outlined by Arksey and O'Malley (Arksey \& O'Malley, 2005). This consists of the following five stages: 
(1) Identifying the research question, (2) Identifying relevant studies, (3) Study selection, (4) Charting the data and (5) Collating, summarizing and reporting the results.

\section{Research Questions}

The overarching review question was: "How do social and structural determinants of health influence outcomes for patients following neurosurgical procedures?" The subreview questions are as follows:

1. What social determinants of health have already been evaluated in neurosurgery?

2. For which procedures/pathologies have these studies been carried out?

\section{Eligibility Criteria}

\section{Participants}

The comprehensive review incorporated studies of pediatric and adult neurosurgical procedures, where pediatric cases are defined as patients less than 18 years old.

\section{Concept}

Any studies where patients were undergoing a neurosurgical procedure, where a social determinant of health was an independent variable in analysis, were included. Further, studies needed to be evaluating differences in post-operative outcomes based on 
the SSDOH, rather than looking at disparities in access to a particular service. Studies comparing surgical outcomes against a nonsurgical control, or those looking at disparities in access to care, were excluded.

\section{Context}

The neurosurgical procedures included in this analysis were delivered in various healthcare settings, both academic and community practices. In addition, there were numerous international studies captured within the search protocol. As the SSDOH are contextual and historically situated, studies conducted outside of the United States were excluded from analysis during the data extraction process.

\section{Search Strategy}

To identify studies for consideration for this systematic review, the review team worked with a medical librarian to develop detailed search strategies for each database. The search was developed for PubMed (NLM) and was translated to Embase (Elsevier), Web of Science (Clarivate Analytics), Cochrane Central (Wiley), and CINAHL (EBSCOHost) using a combination of keywords and subject headings. The PubMed search used a modified version of the MEDLINE/PubMed health disparities search hedge. A grey literature search included ClinicalTrials.gov and National Institute for Health and Care Excellence (NICE). The search was limited to articles published from 
January 1st, 1990 to December 3rd, 2020. The final search was completed on December 3rd, 2020. The full search details are provided as an Appendix.

\section{Citation Management and Study Selection}

All articles retrieved from the database searches were exported and stored in EndNote X.9 bibliographic and reference manager. Studies were screened by title and abstract independently by two reviewers. Any uncertainty about study selection was resolved by an independent third reviewer.

\section{Data Extraction, Collation and Summary}

Extracted data included studied social determinant, first author, publication year, study population, intervention, neurosurgical subspecialty, setting, outcomes, and key findings. Study data were extracted independently and managed using REDCap electronic data capture tools hosted at the authors institution. REDCap (Research Electronic Data Capture) is a secure, web-based software platform designed to support data capture for research studies, providing 1) an intuitive interface for validated data capture; 2) audit trails for tracking data manipulation and export procedures; 3) automated export procedures for seamless data downloads to common statistical packages; and 4) procedures for data integration and interoperability with external sources (Harris et al., 2009; Harris et al., 2019). 
The following tools were utilized for presenting the results of this review: (1) a PRISMA flow diagram detailing the study selection process, (2) figures and tables to display data extracted from eligible studies, (3) a narrative summary detailing the qualified studies in relation to the review questions and objectives. 
Results

Descriptive Numerical Summary

The search resulted in 5,940 studies: 112 from grey literature sources, PubMed (NLM) from 1/1/90 to 12/3/20 (810 Results), Embase (Elsevier) from 1/1/90 to 12/3/20

(1,113 Results), Web of Science (Clarivate Analytics) from 1/1/90 to 12/3/20 (3,092

Results), Cochrane Central (Wiley) from 1/1/90 to 12/3/20 (103 Results), CINAHL (EBSCOHost) from 1/1/90 to 12/3/20 (710 Results). 866 duplicate studies were found and omitted using Endnote X.9 for the deduplication of records and 5,074 references were eligible to screen. After additional screening for duplicate records in Rayyan QCRI, 345 more duplicates were removed, leaving 4,729 references eligible to screen. After title/abstract screening and resolution of studies with conflicting screening decisions, 188 articles remained eligible for review. Bibliographic and citation searching resulted in 4 additional studies being identified, resulting in a total number of 192 studies being included. 38 studies were identified as having taken place outside of the United States and were excluded, resulting in 154 studies remaining. Of the remaining 154, 55 analyses were excluded as they were identified as not explicitly designed with the purpose of evaluating the SSDOH. This resulted in the 99 studies which remained for final inclusion in this review. The selection process from identification to final inclusion is represented 
via a PRISMA flowchart (Figure 1).

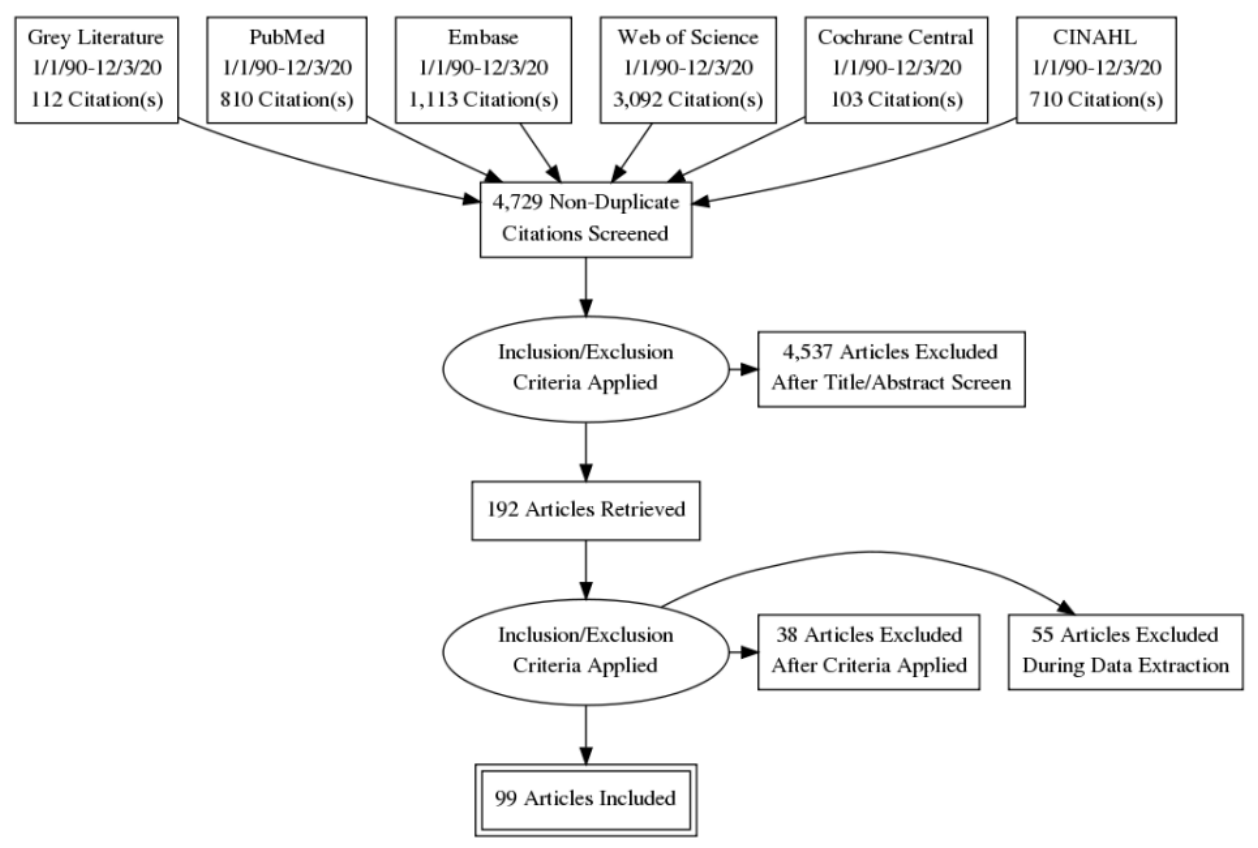

Figure 1. PRISMA Flowchart

\section{Characteristics of Included Studies}

Of the 99 included studies, 6 social determinants were identified, with 3 studies evaluating highest level of educational attainment, 14 studies evaluating gender, 52 studies evaluating race, 41 studies evaluating economic stability, 14 studies evaluating structural determinants (e.g. zip code, provider density, distance to hospital), and 1 study 
evaluating marital status (Table 1, Figure 2).

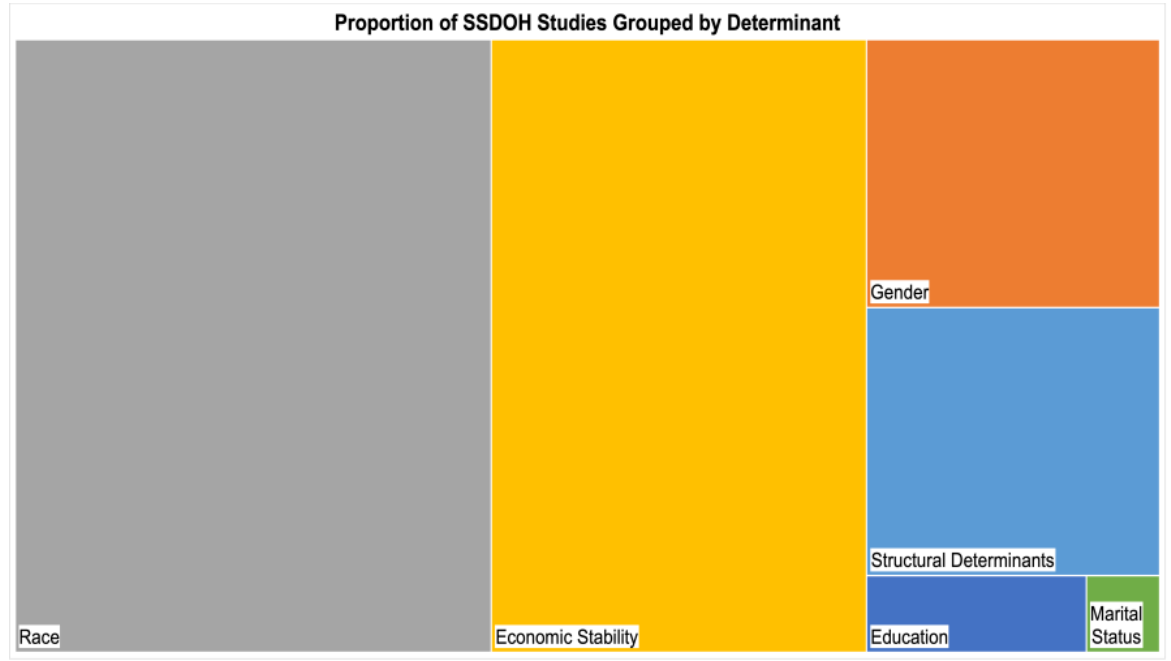

Figure 2. Treemap by SSDOH

With regard to neurosurgical subspecialty, 40 studies were in spine surgery, 4 studies in functional neurosurgery, 14 studies in vascular neurosurgery, 27 studies in cranial oncology, 5 studies in spinal oncology, 5 studies in pediatric neurosurgery, 1 study in trauma and 3 studies in general/unspecified (Table 1, Figure 3).

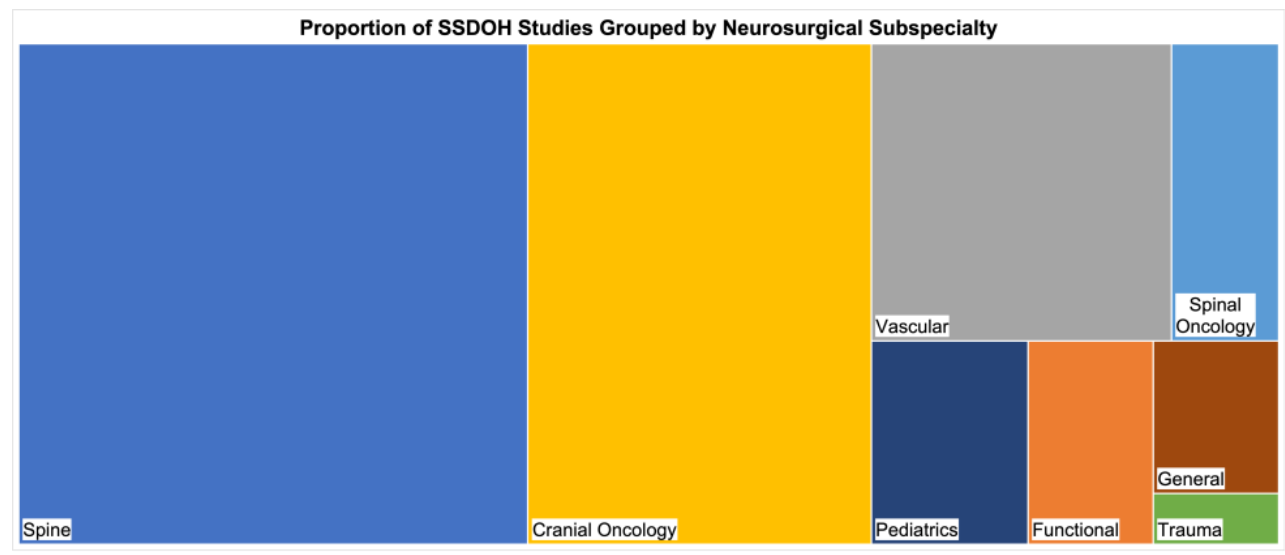

Figure 3. Treemap by Subspecialty

Analyzed outcomes included, but were not limited to, readmissions, quality of life, mortality, complications, overall morbidity and emergency department visits (Table 
2). Chronologically, the first study looking at SSDOH and neurosurgical outcomes took place in 1997, with a trend of increasing studies over time, peaking at 19 in 2020 (Table 1, Figure 4).

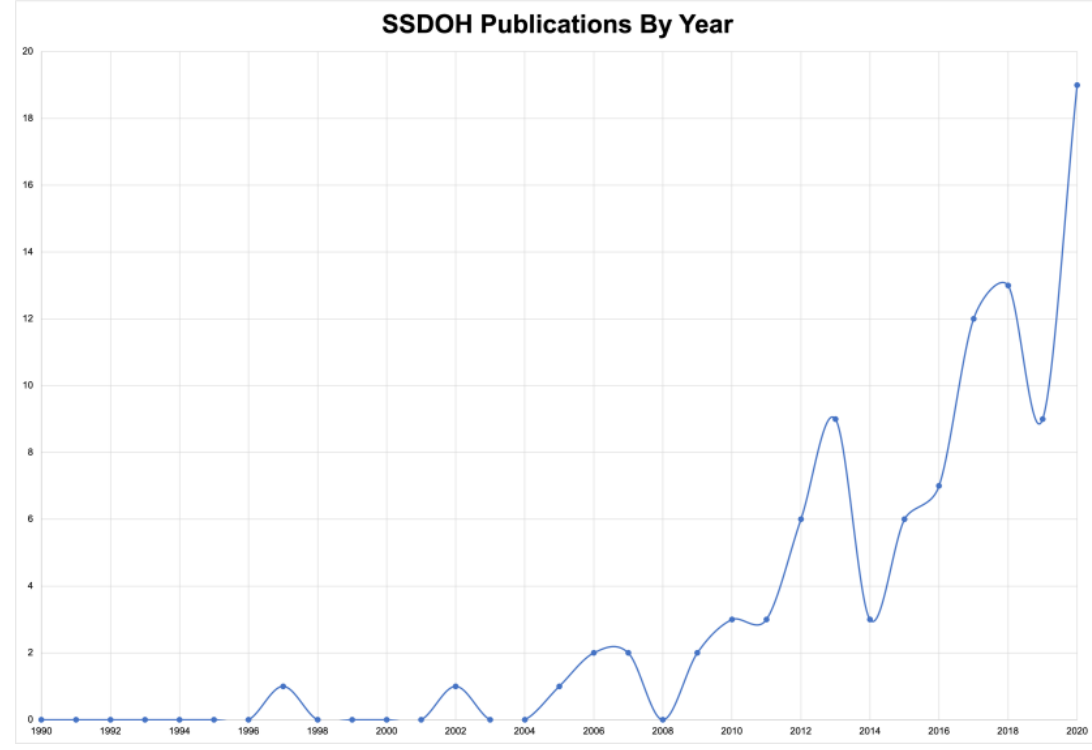

Figure 4. SSDOH Publication Line Graph

Table 1. Characteristics of Included Studies $(n=99)$

\begin{tabular}{|c|c|c|}
\hline Criterion & Characteristic & Number of studies (\%) \\
\hline $\begin{array}{l}\text { Social Determinant } \\
\text { of Health }\end{array}$ & $\begin{array}{l}\text { Education } \\
\text { Gender } \\
\text { Race } \\
\text { Economic Stability } \\
\text { Insurance Status } \\
\text { Employment Status } \\
\text { Socioeconomic Status } \\
\text { Structural Determinants } \\
\text { Marital Status }\end{array}$ & $\begin{array}{l}3 \\
14 \\
52 \\
41 \\
31 \\
3 \\
9 \\
14 \\
1\end{array}$ \\
\hline $\begin{array}{l}\text { Neurosurgical } \\
\text { subspecialty }\end{array}$ & $\begin{array}{l}\text { Spine } \\
\text { Functional } \\
\text { Vascular } \\
\text { Cranial Oncology } \\
\text { Spinal Oncology }\end{array}$ & $\begin{array}{l}40(40.4) \\
4(4.0) \\
14(14.1) \\
27(27.3) \\
5(5.1)\end{array}$ \\
\hline
\end{tabular}


Table 2 continued

\begin{tabular}{|c|c|c|}
\hline & $\begin{array}{l}\text { Pediatrics } \\
\text { Trauma } \\
\text { General/Unspecified }\end{array}$ & $\begin{array}{l}5(5.1) \\
1(1.0) \\
3(3.0)\end{array}$ \\
\hline $\begin{array}{l}\text { Country of origin } \\
\text { of study }\end{array}$ & United States of America & $99(100)$ \\
\hline Year of publication & $\begin{array}{l}1990 \\
1991 \\
1992 \\
1993 \\
1994 \\
1995 \\
1996 \\
1997 \\
1998 \\
1999 \\
2000 \\
2001 \\
2002 \\
2003 \\
2004 \\
2005 \\
2006 \\
2007 \\
2008 \\
2009 \\
2010 \\
2011 \\
2012 \\
2013 \\
2014 \\
2015 \\
2016 \\
2017 \\
2018 \\
2019 \\
2020\end{array}$ & $\begin{array}{l}0(0) \\
0(0) \\
0(0) \\
0(0) \\
0(0) \\
0(0) \\
0(0) \\
1(1.0) \\
0(0) \\
0(0) \\
0(0) \\
0(0) \\
1(1.0) \\
0(0) \\
0(0) \\
1(1.0) \\
2(2.0) \\
2(2.0) \\
0(0) \\
2(2.0) \\
3(3.0) \\
3(3.0) \\
6(6.1) \\
9(9.1) \\
3(3.0) \\
6(6.1) \\
7(7.1) \\
12(12.1) \\
13(13.1) \\
9(9.1) \\
19(19.2)\end{array}$ \\
\hline Population & $\begin{array}{l}\text { Adults only } \\
\text { Children only } \\
\text { Adults and Children }\end{array}$ & $\begin{array}{l}94(94.9) \\
5(5.1) \\
0(0.0)\end{array}$ \\
\hline
\end{tabular}


Table 3. Outcomes Assessed in Included Studies

\begin{tabular}{|l|}
\hline \multicolumn{1}{|c|}{ Outcome Assessed } \\
\hline Readmission \\
Disability Index \\
Return to Work \\
Mortality \\
Quality of life \\
Emergency Department Visits \\
Patient Reported Outcomes \\
Pain Index \\
Discharge disposition \\
Length of Stay \\
Reoperation \\
Functional status \\
Radiographic fusion \\
Discharge to rehab \\
ED evaluation \\
Independent Life \\
Complications \\
Total Costs \\
Hospital Acquired Condition \\
Estimated Blood Loss \\
Morbidity \\
\hline
\end{tabular}

\section{Studied Social Determinants}

\section{Education}

3 studies evaluated the association of education and neurosurgical outcomes (Figure 2). Education was analyzed in 2 studies of spine surgery patients and 1 study in cranial oncology (Figure 5). Specific areas of education that were evaluated were presence/absence of a high school diploma and presence/absence of advanced degrees. 


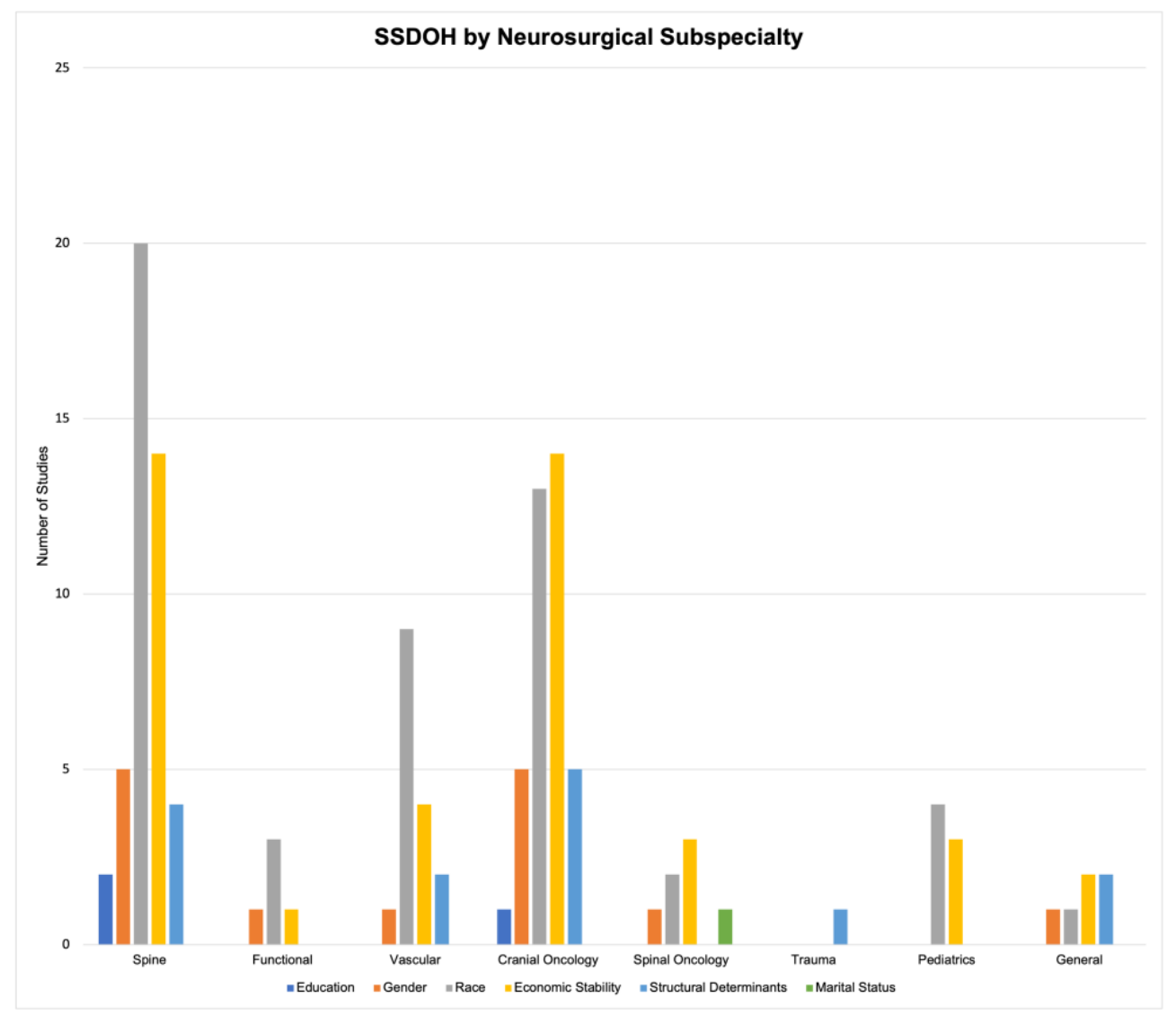

Figure 5. SSDOH by Neurosurgical Subspecialty

\section{Gender}

14 studies evaluated the correlation of gender, categorized in a binary manner (male or female), with neurosurgical outcomes (Figure 2). Gender was analyzed across multiple surgical subspecialties, with 5 studies in spine surgery, 1 study in functional neurosurgery, 1 study in vascular neurosurgery, 5 studies in cranial oncology, 1 study in spinal oncology and 1 study in general/unspecified (Figure 5).

Race 
52 studies evaluated the influence of race on neurosurgical outcomes (Figure 2). While some studies collected data on multiple races (Hispanic/latinx, African-American, White, Asian), the majority of analyses looked at race in a binary manner, comparing outcomes between White and African-American patients. Race was analyzed across multiple surgical subspecialties, with 20 studies in spine surgery, 3 studies in functional neurosurgery, 9 studies in vascular neurosurgery, 13 studies in cranial oncology, 2 studies in spinal oncology, 4 studies in pediatric neurosurgery and 1 study in general/unspecified (Figure 5).

Economic Stability

41 studies analyzed the impact of economic stability on neurosurgical outcomes (Figure 2). Economic stability was analyzed across multiple surgical subspecialties, with 14 studies in spine surgery, 1 study in functional neurosurgery, 4 studies in vascular neurosurgery, 14 studies in cranial oncology, 3 studies in spinal oncology, 3 studies in pediatric neurosurgery and 2 studies in general/unspecified (Figure 5). Economic stability was highly heterogeneous across studies, most often described as insurance status, employment status and household income.

Economic Stability: Insurance Status 
31 studies evaluated the association of insurance status and neurosurgical outcomes. Insurance status was analyzed across multiple surgical subspecialties, with 10 studies in spine surgery, 1 study in functional neurosurgery, 3 studies in vascular neurosurgery, 10 studies in cranial oncology, 2 studies in spinal oncology, 3 studies in pediatric neurosurgery and 2 studies in general/unspecified (Figure 6). Specific areas of insurance status used for analyses were private vs public insurance, Medicaid, self-pay and uncovered.

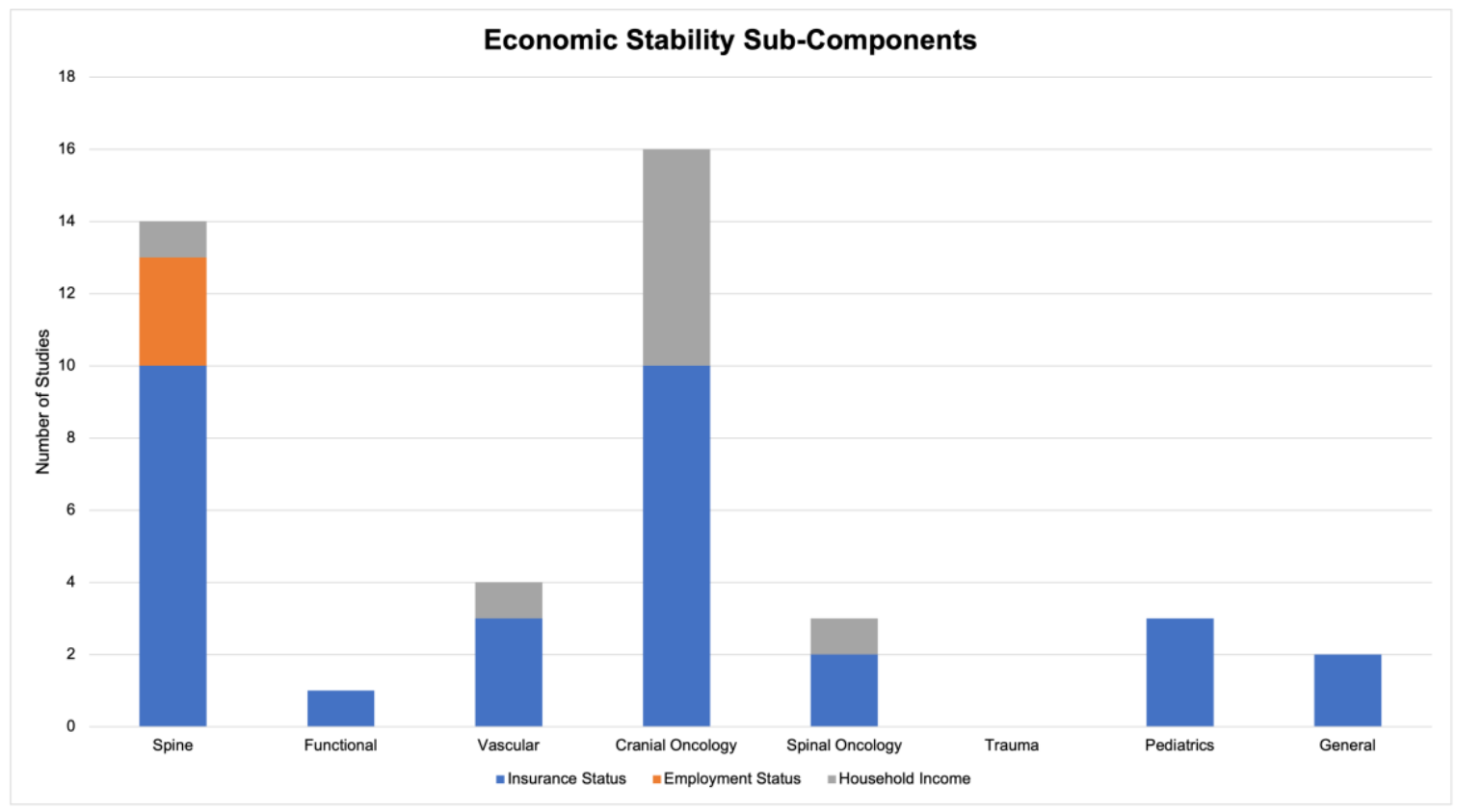

Figure 6. Economic Status Subcomponents

\section{Economic Stability: Employment Status}

3 studies analyzed the influence of employment status on neurosurgical outcomes. Employment status was exclusively analyzed in 3 studies of spine surgery patients 
(Figure 6). Correlates used for employment status included whether patients were on Worker's compensation prior to surgery, if patient's had labor intensive jobs, or if they were employed pre-operatively.

Economic Stability: Household Income

9 studies assessed the correlation of household income and neurosurgical outcomes. Household income was analyzed across multiple surgical subspecialties, with 1 study in spine surgery, 1 study in vascular neurosurgery, 6 studies in cranial oncology and 1 study in spinal oncology (Figure 6). Studies evaluating household income stratified their study population into income quartiles based on the median income in their zip code, analyzing outcome differences between low income quartile and high income quartile patients.

\section{Structural Determinants}

14 studies evaluated the impact of structural determinants on neurosurgical outcomes (Figure 2). Studies that evaluated structural determinants looked at patient zip code, distance to a high-volume academic medical center and density of neurosurgical providers in the patient's area. Structural determinants were analyzed across multiple surgical subspecialties, with 4 studies in spine surgery, 2 studies in vascular 
neurosurgery, 5 studies in cranial oncology, 1 study in trauma and 2 studies in general/unspecified (Figure 5).

\section{Marital Status}

1 study assessed the influence of marital status on neurosurgical outcomes (Figure 2). Marital status was evaluated exclusively in spinal oncology, comparing outcomes between married and non-married patients (Figure 5). 


\title{
CHAPTER 3
}

\section{STRUCTURAL AND SOCIAL DETERMINANTS OF HEALTH IN NEUROSURGERY}

\author{
Summary of Results
}

This scoping review is the first to identify the extent of studies evaluating SSDOH in neurosurgery. To date, studies in neurosurgery have found that low-income patients and African-american patients are at increased risk of post-operative mortality following brain tumor resection (Glauser, Dimentberg, Shultz, McClintock, \& Malhotra, 2020; Haldar, Glauser, Winter, Goodrich et al., 2020). Further, prior work has found that there are several racial disparities in length of stay, costs and rate of complications after surgery for lumbar stenosis and other elective spine procedures (Lad et al., 2013; Seicean, Seicean, Neuhauser, Benzel, \& Weil, 2017). These data suggest that the SSDOH are relevant factors for neurosurgical outcomes.

The literature regarding how the SSDOH impact neurosurgical outcomes is expanding. Notably, the majority of studies were published in the 2010's (91\%), increasing steadily throughout the decade and peaking in 2020 (Table 1, Figure 4). A large proportion of studies evaluated gender, race and economic stability, with 14 studies evaluating gender, 52 analyzing race and 41 assessing economic stability. Less studies evaluated the impact of structural determinants and marital status on neurosurgical outcomes. 
Further, no studies directly assessed the multitude of other SSDOH, including social and community context (social cohesion, incarceration, etc.), health literacy, food insecurity and housing instability, among others. There are various potential causes for the lack of studies in these areas. Prior to 2020, SSDOH were not explicity included in patient records (New ICD-10 codes will help physicians tackle social barriers to care.2019). Demographic information, such as patient gender, race, and insurance status have traditionally been collected. In addition, details about a patient's employment status is often collected, particularly since that information may be relevant for spine surgery (e.g. heavy lifting at work for a patient with low-back pain). Household income, while typically not directly collected, can be extrapolated from patient zip code data for research purposes. Inquiring about factors such as social context, health literacy, and food insecurity were rarely deemed as directly relevant to a patients neurosurgical condition. Furthermore, neurosurgical training has traditionally not emphasized holistic views of patient care. Therefore, the SSDOH that move beyond traditional demographics (e.g. race, biological gender, education and insurance status as proxies for class) were likely not considered as relevant for neurosurgical patients.

Of note, numerous studies focused on the SSDOH in neurosurgery align with the principals laid out in the position statements of the AAFP, Society of General Internal Medicine and the American College of Physicians. These groups each emphasize a need for increased use of healthcare data to drive studies evaluating how the SSDOH impact patients. Multiple studies evaluating race and household income for patients undergoing brain tumor resection utilized data extraction tools which were built in to the electronic health record at the authors institution (Glauser et al., 2020; Haldar et al., 2020; Haldar, 
Glauser, Winter, Dimentberg et al., 2020). A number of studies looking at the SSDOH in spine surgery made use of data from nationally maintained databases, which sourced information from participating institutions electronic health records (Lad et al., 2013; Rethorn, Garcia, Cook, \& Gottfried, 2020). These examples elucidate that neurosurgeons are considering the same principles set out by primary care groups when designing studies to evaluate the SSDOH. Although a number of research gaps were identified by this scoping review, the aforementioned examples suggest that some neurosurgeons are beginning to incorporate the SSDOH in their work.

\section{Identified Research Gaps}

There are multiple research gaps identified through this thesis. While gender, race and economic stability were analyzed in a comparatively robust manner, there remains a paucity of literature surrounding the other SSDOH in this review (Table 3).

Table 4. Studied SSDOH by Neurosurgical Subspecialty

$$
\begin{array}{llllllll}
\text { Spine Functional Vascular } & \begin{array}{l}
\text { Cranial } \\
\text { Oncology }
\end{array} & \begin{array}{l}
\text { Spinal Trauma } \\
\text { Oncology }
\end{array} & \text { Pediatrics General Total }
\end{array}
$$

\begin{tabular}{l|l|l|l|l|l|l|l|l|l}
\hline Education & 2 & 0 & 0 & 1 & 0 & 0 & 0 & 0 & 3 \\
\hline Gender & 5 & 1 & 1 & 5 & 1 & 0 & 0 & 1 & 14 \\
\hline Race & 20 & 3 & 9 & 13 & 2 & 0 & 4 & 1 & 52 \\
\hline $\begin{array}{l}\text { Economic } \\
\text { Status }\end{array}$ & 14 & 1 & 4 & 14 & 3 & 0 & 3 & 2 & 41 \\
\hline Structural & 4 & 0 & 2 & 5 & 0 & 1 & 0 & 2 & 14 \\
\hline $\begin{array}{l}\text { Marital } \\
\text { status }\end{array}$ & 0 & 0 & 0 & 0 & 1 & 0 & 0 & 0 & 1 \\
\hline Total & 45 & 5 & 16 & 38 & 7 & 1 & 7 & 6 & 125 \\
\hline
\end{tabular}


Further, the volume of literature evaluating the SSDOH in comparison to the overall neurosurgical literature remains low. While the trend over the 2010's of increasing studies looking at the SSDOH may be encouraging, it is important to note that overall academic publishing - and publishing in neurosurgery - have followed similar trends (Harsh, Adnan, Raees, Manjul, \& Anil, 2021; Matthews, Yang, Lewis, Vaidyanathan, \& Gorman, 2020). Hence, it is challenging to assess whether the growth of studies on the SSDOH in neurosurgery is the result of a growing value placed on these topics in the field or secondary to a growing volume of research output.

Another research gap identified by this thesis is that the majority of the 99 included analyses of SSDOH in neurosurgery are focused on spine surgery patients. The breakdown of number of studies is similar to the percentage of neurosurgical procedures performed, as typical neurosurgery departments have $60-70 \%$ of their volume from spine surgery (Smith, 2017). In addition, clinical research in spine surgery has been at the forefront of neurosurgical specialties with respect to outcomes assessment. This is due to the concern that spine surgery carries higher costs and incidences of harm, which has driven a large amount of effort towards modeling ideal candidates for surgery (Rethorn et al., 2020). Between the high volume of cases and focus on outcomes research, it is unsurprising that the majority of SSDOH studies in neurosurgery are spine focused. Nevertheless, only two of the remaining subspecialties have greater than 10 studies on the SSDOH (cranial oncology and vascular), with the remaining subspecialties - functional, spinal oncology, trauma, pediatrics - falling below that number. The low number of studies of the SSDOH in the majority of neurosurgical subspecialties presents a strong opportunity for future research. 


\section{Future Directions}

Many of the outcome measures found in the included studies are those used by the Centers for Medicare \& Medicaid Services (CMS) as quality metrics. This thesis found that SSDOH were tied to numerous clinical outcomes, including readmission, emergency department visits, reoperation, mortality, quality of life, return to work, discharge disposition and length of stay, amongst others. In addition to the CMS quality measures, these outcomes have substantial financial and clinical significance.

Research into each of the SSDOH may help decision-makers to identify at-risk patients and drive initiatives to target outcome improvement. As the SSDOH are coded in the health record moving forward, there will be abundant opportunity for research and improved patient care. A novel method of merging the SSDOH for the purpose of risk stratification is the development of a polysocial risk score, as described by Figueroa et al (Figueroa, Frakt, \& Jha, 2020). This polysocial risk score would account for the fact that the SSDOH are interrelated and complex. Further, the polysocial risk score provides a data-driven, renewed way of thinking about the SSDOH (Figueroa et al., 2020). Establishment of efforts such as a polysocial risk score requires extensive individuallevel information on social determinants and needs, all of which must be linked to patients' health status and outcomes (Figueroa et al., 2020). An elevated amount of data and potential tools, such as a polysocial risk score, can be utilized between neurosurgeons and their associated care teams (social work, patient outreach, care coordination, etc.) to enact efforts for outcome equity. 
The present scoping review is the first to evaluate the SSDOH in a neurosurgical population. This review was conducted in a comprehensive manner, encompassing a search of five electronic databases of peer-reviewed literature and a wide array of grey literature sources. Further, this search was not susceptible to geographical bias, as the review was not restricted to studies only in English. Hence, this review offers a holistic assessment of how the SSDOH have been studied in neurosurgery to date. As this scoping review was performed with a well-established, thorough review protocol with a systematic search strategy, the ease of replication and reliability of this thesis are high.

\section{Limitations}

While this review covered the breadth of evidence available regarding the $\mathrm{SSDOH}$ in neurosurgery, this thesis is not without limitations. As an extensive range of studies were included in the review, there remains the possibility that studies with weak methodology were incorporated. In addition, quality assessment of all of the included studies was not performed by the authors. However, the goal of this review was to evaluate the extent of SSDOH literature in neurosurgery, without assessing article quality or the reliability of reported outcomes.

\section{Conclusion}


This scoping review of the SSDOH in neurosurgical outcomes identified 99 studies from the year 1990 to 2020. Identified studies were targeted predominantly toward spine surgery patients, evenly distributed in analyses of gender, race and economic stability. Research gaps include the remaining neurosurgical subspecialties and numerous other SSDOH. These gaps should be areas of future study, with the goal of aligning research with new healthcare initiatives and assuring that the SSDOH are extensively accounted for to optimize neurosurgical care. The SSDOH align with the guiding principles of medical ethics and are relevant for all practicing physicians, regardless of medical or surgical specialty. The relatively low volume of neurosurgical papers focused on the SSDOH and proportion of papers in one subspecialty demonstrates the need for an expanded interest in the SSDOH in neurosurgery. 


\section{REFERENCES CITED}

Advancing health equity by addressing the social determinants of health in family medicine (position paper). Retrieved from

https://www.aafp.org/about/policies/all/social-determinants-health-familymedicine.html

Arksey, H., \& O'Malley, L. (2005). Scoping studies: Towards a methodological framework. International Journal of Social Research Methodology, 8(1), 19-32. doi:10.1080/1364557032000119616

Blacksher, E., \& Lovasi, G. S. (2012). Place-focused physical activity research, human agency, and social justice in public health: Taking agency seriously in studies of the built environment. Health \& Place, 18(2), 172-179.

doi:10.1016/j.healthplace.2011.08.019

Blustein, J., \& Fleischman, A. R. (2004). Urban bioethics: Adapting bioethics to the urban context. Academic Medicine: Journal of the Association of American Medical Colleges, 79(12), 1198-1202. doi:10.1097/00001888-200412000-00013

Byhoff, E., Kangovi, S., Berkowitz, S. A., DeCamp, M., Dzeng, E., Earnest, M., . . DeSalvo, K. (2020). A society of general internal medicine position statement on the 
internists' role in social determinants of health. Journal of General Internal Medicine, 35(9), 2721-2727. doi:10.1007/s11606-020-05934-8

Catalyst, N. (2017). Social determinants of health (SDOH). NEJM Catalyst, Retrieved from https://catalyst.nejm.org/doi/full/10.1056/CAT.17.0312

Daniel, H., Bornstein, S. S., \& Kane, G. C. (2018a). Addressing social determinants to improve patient care and promote health equity: An american college of physicians position paper. Annals of Internal Medicine, 168(8), 577-578. doi:10.7326/M172441

Figueroa, J. F., Frakt, A. B., \& Jha, A. K. (2020). Addressing social determinants of health: Time for a polysocial risk score. Jama, doi:10.1001/jama.2020.2436

Glauser, G., Dimentberg, R., Shultz, K., McClintock, S. D., \& Malhotra, N. R. (2020). Survival disparity based on household income in 1970 patients following brain tumor surgery. World Neurosurgery, 143, e112-e121. doi:10.1016/j.wneu.2020.07.016

Haldar, D., Glauser, G., Winter, E., Dimentberg, R., Goodrich, S., Shultz, K., . . . Malhotra, N. R. (2020). The effect of race on short-term pituitary tumor outcomes. World Neurosurgery, 137, e447-e453. doi:10.1016/j.wneu.2020.01.241

Haldar, D., Glauser, G., Winter, E., Goodrich, S., Shultz, K., McClintock, S. D., \& Malhotra, N. R. (2020). Assessing the role of patient race in disparity of 90-day 
brain tumor resection outcomes. World Neurosurgery, doi:10.1016/j.wneu.2020.04.098

Harris, P. A., Taylor, R., Minor, B. L., Elliott, V., Fernandez, M., O'Neal, L., . . Duda, S. N. (2019). The REDCap consortium: Building an international community of software platform partners. Journal of Biomedical Informatics, 95, 103208. doi:10.1016/j.jbi.2019.103208

Harris, P. A., Taylor, R., Thielke, R., Payne, J., Gonzalez, N., \& Conde, J. G. (2009). Research electronic data capture (REDCap)--a metadata-driven methodology and workflow process for providing translational research informatics support. Journal of Biomedical Informatics, 42(2), 377-381. doi:10.1016/j.jbi.2008.08.010

Harsh, D., Adnan, H. S., Raees, A. P., Manjul, T., \& Anil, N. (2021). How many neurosurgeons does it take to author an article and what are the other factors that impact citations? World Neurosurgery, 146, e993-e1002. doi:10.1016/j.wneu.2020.11.058

Lad, S. P., Bagley, J. H., Kenney, K. T., Ugiliweneza, B., Kong, M., Bagley, C. A., . . Boakye, M. (2013). Racial disparities in outcomes of spinal surgery for lumbar stenosis. Spine, 38(11), 927-935. doi:10.1097/BRS.0b013e31828165f9

Maani, N., \& Galea, S. (2020). The role of physicians in addressing social determinants of health. Jama, doi:10.1001/jama.2020.1637 
Mapping life expectancy: Philadelphia. (2016). Retrieved from https://societyhealth.vcu.edu/work/the-projects/mapsphiladelphia.html

Matthews, K. R. W., Yang, E., Lewis, S. W., Vaidyanathan, B. R., \& Gorman, M. (2020). International scientific collaborative activities and barriers to them in eight societies. Accountability in Research, 27(8), 477-495. doi:10.1080/08989621.2020.1774373

McCormick, T. R.Principles of bioethics. (2018). Retrieved from https://depts.washington.edu/bhdept/ethics-medicine/bioethics$\underline{\text { topics/articles/principles-bioethics }}$

New ICD-10 codes will help physicians tackle social barriers to care. (2019). Retrieved from https://www.ama-assn.org/practice-management/digital/new-icd-10-codes-willhelp-physicians-tackle-social-barriers-care

Rethorn, Z. D., Garcia, A. N., Cook, C. E., \& Gottfried, O. N. (2020). Quantifying the collective influence of social determinants of health using conditional and cluster modeling. PloS One, 15(11), e0241868. doi:10.1371/journal.pone.0241868

Seicean, A., Seicean, S., Neuhauser, D., Benzel, E. C., \& Weil, R. J. (2017). The influence of race on short-term outcomes after laminectomy and/or fusion spine surgery. Spine, 42(1), 34-41. doi:10.1097/BRS.0000000000001657

Smedley, Brian D., Stith, Adrienne Y., Nelson, Alan R. (2003). In Smedley B. D., Stith A. Y. and Nelson A. R. (Eds.), Unequal treatment: Confronting racial and ethnic 
disparities in health care. Washington (DC): National Academies Press (US). Retrieved from http://www.ncbi.nlm.nih.gov/books/NBK220358/

Smith, D. A. (2017, -08-16T09:00:28\&\#43;00:00). Why you should choose a neurosurgeon over an orthopedic surgeon. Retrieved from https://rockymountainbrainandspineinstitute.com/choose-neurosurgeon-orthopedicsurgeon/

Statement on optimal access. (2013). Retrieved from https://www.facs.org/aboutacs/statements/72-optimal-access

Torain, M. J., BS, Maragh-Bass, Allysha C., PhD, MPH, Dankwa-Mullen, Irene, MD, MPH, Hisam, B., MD, Kodadek, L. M., MD, Lilley, Elizabeth J., MD, MPH, . . . Haider, Adil H., MD, MPH, FACS. (2015). Surgical disparities: A comprehensive review and new conceptual framework. Journal of the American College of Surgeons, 223(2), 408-418. doi:10.1016/j.jamcollsurg.2016.04.047

Tricco, A. C., Lillie, E., Zarin, W., O'Brien, K. K., Colquhoun, H., Levac, D., . . Straus, S. E. (2018). PRISMA extension for scoping reviews (PRISMA-ScR): Checklist and explanation. Annals of Internal Medicine, 169(7), 467-473. doi:10.7326/M18-0850

West, M. A., Hwang, S., Maier, R. V., Ahuja, N., Angelos, P., Bass, B. L., .. Wren, S. M. (2018). Ensuring equity, diversity, and inclusion in academic surgery: An american surgical association white paper. Annals of Surgery, 268(3), 403-407. doi:10.1097/SLA.0000000000002937 
World Health Assembly, 62. (2009). Reducing health inequities through action on the social determinants of health. Geneva: World Health Organization. Retrieved from https://apps.who.int/iris/handle/10665/2257 


\section{APPENDIX: FULL SEARCH PROTOCOL}

PubMed (NLM)

("ethnic disparity"[TIAB] OR "ethnic disparities"[tiab] OR "health disparit*"[TIAB]

OR "healthcare disparities"[MH] OR "health care disparity"[TIAB] OR "health care disparities"[TIAB] OR "healthcare disparity"[TIAB] OR "healthcare disparities"[TIAB] OR "health-care disparity"[TIAB] OR "health-care disparities"[TIAB] OR "health status disparities"[MH] OR "disparity in health"[TIAB] OR "disparities in health"[TIAB] OR "culturally competent care"[MH] OR "culturally competent care"[TIAB] OR "delivery of health care"[MH:noexp] OR "disparity"[TIAB] OR "disparities"[TIAB] OR "health related quality of life"[TIAB] OR "health-related quality of life"[TIAB] OR "health services accessibility" $[\mathrm{MH}]$ OR "health services, indigenous" $[\mathrm{MH}]$ OR "health services needs and demand"[MH] OR "health status disparities"[MH] OR "patient acceptance of health care" $[\mathrm{MH}]$ OR "patient selection"[MH] OR "quality of health care"[MeSH Major Topic:noexp] OR "social class"[MH] OR "social class"[TIAB] OR "social classes"[TIAB] OR "social norm"[TIAB] OR "social norms"[TIAB] OR "social determinants of health"[MH] OR "social determinants of health"[TIAB] OR "social disparity"[TIAB] OR "social disparities"[TIAB] OR "social factor"[TIAB] OR "social factors"[TIAB] OR "social inequity"[TIAB] OR "social inequities"[TIAB] OR "socioeconomic factor"[TIAB] OR "socioeconomic factors"[TIAB] OR "socioeconomic factors"[MH] OR "socioeconomically disadvantaged"[TIAB] OR "zip code"[TIAB] OR educat*[TIAB] OR "education level"[TIAB] OR "health literacy"[MH] OR "health literacy"[TIAB] OR "economic stability"[TIAB] OR "household income"[TIAB] OR "food supply"[MH] OR "food insecurity"[TIAB]) AND ("African American"[TIAB] OR "African Americans"[TIAB] OR "African ancestry"[TIAB] OR "ageism"[MH] OR AIAN[TIAB] OR "Alaska Native"[TIAB] OR Asian*[TIAB] OR "Black American"[TIAB] OR "Black Americans"[TIAB] OR Caucasian*[TIAB] OR disabled[TIAB] OR "disabled persons"[MH] OR "disabled persons"[TIAB] OR "diverse population"[TIAB] OR "diverse populations"[TIAB] OR "emigrants and immigrants" $[\mathrm{MH}]$ OR "ethnic group"[TIAB] OR "ethnic groups"[TIAB] OR "ethnic groups"[MH] OR "ethnic inequality"[TIAB] OR "ethnic inequalities"[TIAB] OR "ethnic population"[TIAB] OR "ethnic populations"[TIAB] OR ghetto*[TIAB] OR "health services for persons with disabilities"[MH] OR Hispanic*[TIAB] OR "hispanic americans"[MH] OR homeless[TIAB] OR "homeless persons"[MH] OR immigrant*[TIAB] OR Indian*[TIAB] OR "indians, north american"[MH] OR inmate*[TIAB] OR jail*[TIAB] OR "jail population"[TIAB] OR "jail populations"[TIAB] OR Latina*[TIAB] OR Latino*[TIAB] OR "mexican americans"[MH] OR "medically underserved area" $[\mathrm{MH}]$ OR "medically uninsured"[MH] OR "minorities health"[TIAB] OR "minority group"[TIAB] OR "minority groups"[TIAB] OR "minority groups" $[\mathrm{MH}] \mathrm{OR}$ "minority health" $[\mathrm{MH}]$ OR "minority health"[TIAB] OR "minority population"[TIAB] OR "minority populations"[TIAB] OR "migrant worker"[TIAB] OR "migrant workers"[TIAB] OR "Native American"[TIAB] OR "Native Americans"[TIAB] OR "Pacific Islander"[TIAB] OR "Pacific Islanders"[TIAB] OR "people of color"[TIAB] OR "poverty"[MH] OR poverty[TIAB] OR "poverty areas"[MH] OR "poverty area"[TIAB] OR "poverty areas"[TIAB] OR prisoner*[TIAB] OR "prisoners"[MH] OR "race factors"[MH] OR "race factor"[TIAB] OR "race factors"[TIAB] OR "race and ethnicity"[TIAB] OR "racial and ethnic minority"[TIAB] OR "racial and ethnic minorities"[TIAB] OR "racial 
discrimination"[TIAB] OR "racial disparity"[TIAB] OR "racial disparities"[TIAB] OR "racial equality"[TIAB] OR "racial equity"[TIAB] OR "racial inequity"[TIAB] OR "racial inequities"[TIAB] OR "racial prejudice"[TIAB] OR "racial segregation"[TIAB] OR "racism" [MH] OR "refugees"[MH] OR refugee*[TIAB] OR "rural health"[MH] OR "rural health"[TIAB] OR "rural health services"[MH] OR "rural population"[MH] OR "rural population"[TIAB] OR "rural populations"[TIAB] OR "sexism"[MH] OR slum*[TIAB] OR "social discrimination"[MH] OR "social marginalization"[MH] OR "social segregation"[MH] OR "transients and migrants"[MH] OR underserved[TIAB] OR "undocumented immigrants"[MH] OR "medically uninsured"[MH] OR uninsured[TIAB] OR "urban health"[MH] OR "urban health services"[MH] OR "urban population"[MH] OR "urban population"[TIAB] OR "urban populations"[TIAB] OR "vulnerable population"[TIAB] OR "vulnerable populations"[TIAB] OR "vulnerable populations"[MH] OR bisexual*[TIAB] OR gay[TIAB] OR gays[TIAB] OR "gender change"[TIAB] OR "gender dysphoria"[TIAB] OR "gender diverse"[TIAB] OR "gender diversity"[TIAB] OR "gender-diverse"[TIAB] OR "gender identity"[MH] OR "gender identity"[TIAB] OR "gender identities"[TIAB] OR "gender minority"[TIAB] OR "gender minorities"[TIAB] OR "gender non conforming"[TIAB] OR "gender non-conforming"[TIAB] OR "gender orientation"[TIAB] OR genderqueer[TIAB] OR "gender reassignment"[TIAB] OR "gender surgery"[TIAB] OR "gender surgeries"[TIAB] OR GLBT*[TIAB] OR LGBT*[TIAB] OR "health services for transgender persons"[MH] OR homophil*[TIAB] OR homosexual*[TIAB] OR "homosexuality"[MH] OR "homosexuality, female"[MH] OR "homosexuality, male"[MH] OR intersex[TIAB] OR lesbian*[TIAB] OR "men having sex with men"[TIAB] OR "men who have sex with men"[TIAB] OR "men who have sex with other men"[TIAB] OR nonheterosexual*[TIAB] OR "non-heterosexual"[TIAB] OR "non-heterosexuals"[TIAB] OR "non heterosexual"[TIAB] OR "non heterosexuals"[TIAB] OR pansexual[TIAB] OR polysexual[TIAB] OR "queer"[All Fields] OR "same sex"[TIAB] OR "sexual and gender disorders" $[\mathrm{MH}]$ OR "sexual and gender minorities"[MH] OR "sex change"[TIAB] OR "sex reassignment"[TIAB] OR "sexual diversity"[TIAB] OR "sexual minority"[TIAB] OR "sexual minorities"[TIAB] OR "sexual orientation"[TIAB] OR transgender*[TIAB] OR "transgender persons"[MH] OR transsexual*[TIAB] OR transman[TIAB] OR "trans men"[TIAB] OR transmen[TIAB] OR "transsexualism" $[\mathrm{MH}]$ OR transsexualism[TIAB] OR transwoman[TIAB] OR "trans women"[TIAB] OR transwomen[TIAB] OR inequit*[TIAB] OR "Insurance"[MH] OR insuranc*[TIAB] OR "socioeconomic index"[TIAB] OR "socioeconomic indexes"[TIAB] OR "marital status"[MH] OR "marital status"[TIAB] OR gender*[TIAB])

AND ("Neurosurgery"[Mesh] OR "Neurosurgical Procedures"[Mesh] OR neurosurg*[TIAB] OR "Spinal Cord Neoplasms"[Mesh] OR "Spinal Neoplasms"[Mesh] OR "Nervous System Neoplasms"[Mesh] OR ((spinal[TIAB] OR spine[TIAB] OR nervous[TIAB] OR nerve[TIAB]) AND (neoplasm*[TIAB] OR tumor*[TIAB] OR tumour*[TIAB] OR surg*[TIAB]))) AND (1990:2020[pdat]) AND (“retrospective studies”[MESH] OR "retrospective stud*"[TIAB] OR (retrospective[TIAB] AND stud*[TIAB]) OR "Randomized Controlled Trial" [Publication Type] OR "RCT"[TIAB] OR (random*[TIAB] AND control*[TIAB] AND trial*[TIAB]) OR "Random Allocation"[MESH] OR (random*[TIAB] and allocat*[TIAB]) OR "cohort studies"[MESH] OR (cohort[TIAB] AND stud*[TIAB]) OR (outcom*[TIAB] AND analy*[TIAB]) OR "Case-Control Studies"[MESH] OR "case control stud*”[TIAB] OR “case-control stud*”[TIAB] OR (case[TIAB] AND control[TIAB] AND 
stud*[TIAB]) OR "Case Reports" [Publication Type] OR "case report*”[TIAB] OR (case[TIAB] AND report*[TIAB]) OR "case series"[TIAB] OR (case[TIAB] AND series[TIAB]))

Embase (Elsevier)

('ethnic disparit*':ab,ti OR 'health disparit*':ab,ti OR 'health care disparity'/exp OR 'health care disparit*':ab,ti OR 'healthcare disparit*':ab,ti OR 'health-care disparit*':ab,ti OR 'health disparity'/exp OR 'disparit* in health':ab,ti OR 'transcultural care'/exp OR 'culturally competent care':ab,ti OR 'health care delivery'/de OR 'disparit*':ab,ti OR 'health related quality of life':ab,ti OR 'health-related quality of life':ab,ti OR 'health care access'/de OR 'indigenous health care'/exp OR 'patient attitude'/de OR 'patient selection'/exp OR 'health care quality'/de OR 'social class'/exp OR 'social class*':ab,ti OR 'social norm*':ab,ti OR 'social determinants of health'/exp OR 'social determinants of health':ab,ti OR 'social disparit*':ab,ti OR 'social factor*':ab,ti OR 'social inequit*':ab,ti OR 'socioeconomic factor*':ab,ti OR 'socioeconomics'/exp OR 'socioeconomically disadvantaged':ab,ti OR 'zip code':ab,ti OR educat*:ab,ti OR 'education level':ab,ti OR 'health literacy'/exp OR 'health literacy':ab,ti OR 'economic stability':ab,ti OR 'household income':ab,ti OR 'food supply'/exp OR 'food supply':ab,ti OR 'food insecur*':ab,ti) AND ('African American*':ab,ti OR 'African ancestry':ab,ti OR 'ageism'/exp OR AIAN:ab,ti OR 'Alaska Native*':ab,ti OR Asian*:ab,ti OR 'Black American*':ab,ti OR Caucasian*:ab,ti OR disabled:ab,ti OR 'disabled person'/exp OR 'disabled person*':ab,ti OR 'diverse population*':ab,ti OR 'migrant'/exp OR 'ethnic group*':ab,ti OR 'ethnic group'/exp OR 'ethnic inequality':ab,ti OR 'ethnic inequalit*':ab,ti OR 'ethnic population*':ab,ti OR ghetto*:ab,ti OR Hispanic*:ab,ti OR 'Hispanic'/exp OR homeless*:ab,ti OR 'homeless person'/exp OR immigrant*:ab,ti OR Indian*:ab,ti OR 'American Indian'/exp OR inmate*:ab,ti OR jail*:ab,ti OR 'jail population*':ab,ti OR Latina*:ab,ti OR Latino*:ab,ti OR 'Mexican American'/exp OR 'health care planning'/exp OR 'medically uninsured'/exp OR 'minorit* health':ab,ti OR 'minority group*':ab,ti OR 'minority group'/exp OR 'minority health'/exp OR 'minority health':ab,ti OR 'minority population*':ab,ti OR 'migrant worker*':ab,ti OR 'Native American*':ab,ti OR 'Pacific Islander*':ab,ti OR 'people of color':ab,ti OR 'poverty'/exp OR poverty:ab,ti OR 'poverty area*':ab,ti OR prisoner*:ab,ti OR 'prisoner'/exp OR 'race'/exp OR 'race factor*':ab,ti OR 'race and ethnicity':ab,ti OR 'racial and ethnic minorit*':ab,ti OR 'racial discrimination':ab,ti OR 'racial disparit*':ab,ti OR 'racial equality':ab,ti OR 'racial equity':ab,ti OR 'racial inequit*':ab,ti OR 'racial prejudic*':ab,ti OR 'racial segregation':ab,ti OR 'racism'/exp OR 'refugee'/exp OR refugee*:ab,ti OR 'rural health'/exp OR 'rural health care'/exp OR 'rural health':ab,ti OR 'rural population'/exp OR 'rural population*':ab,ti OR 'sexism'/exp OR slum*:ab,ti OR 'social discrimination'/exp OR 'social exclusion'/exp OR 'social segregation'/exp OR 'migration'/exp OR underserved:ab,ti OR 'undocumented immigrant'/exp OR uninsured:ab,ti OR 'urban 
health'/exp OR 'urban population'/exp OR 'urban population*':ab,ti OR 'vulnerable population*':ab,ti OR 'vulnerable populations'/exp OR bisexual*:ab,ti OR gay:ab,ti OR gays:ab,ti OR 'gender change':ab,ti OR 'gender dysphoria':ab,ti OR 'gender divers*':ab,ti OR 'gender-diverse':ab,ti OR 'gender identity'/exp OR 'gender identit*':ab,ti OR 'gender minorit*':ab,ti OR 'gender non conform*':ab,ti OR 'gender orientation':ab,ti OR genderqueer:ab,ti OR 'gender queer':ab,ti OR 'gender reassignment':ab,ti OR 'gender surger*':ab,ti OR GLBT:ab,ti OR GLBTQ:ab,ti OR homophil*:ab,ti OR homosexual*:ab,ti OR 'homosexuality'/exp OR 'male homosexuality'/exp OR 'lesbianism'/exp OR 'intersex*':ab,ti OR lesbian*:ab,ti OR LGBBTQ:ab,ti OR LGBT:ab,ti OR LGBTI:ab,ti OR LGBTQ:ab,ti OR LGBTQI:ab,ti OR LGBTQIA:ab,ti OR 'men having sex with men':ab,ti OR 'men who have sex with men':ab,ti OR 'men who have sex with other men':ab,ti OR nonheterosexual*aab,ti OR 'non-heterosexual':ab,ti OR 'non-heterosexuals':ab,ti OR 'non heterosexual*':ab,ti OR pansexual*:ab,ti OR polysexual*:ab,ti OR queer:ab,ti OR 'same sex*':ab,ti OR 'sexual dysfunction'/exp OR 'sexual and gender minority'/exp OR 'sex change':ab,ti OR 'sex reassignment':ab,ti OR 'sexual diversit*':ab,ti OR 'sexual minorit*':ab,ti OR 'sexual orientation*':ab,ti OR transgender**:ab,ti OR 'transgender'/exp OR transsexual*:ab,ti OR transman:ab,ti OR 'trans men':ab,ti OR transmen:ab,ti OR 'transsexualism'/exp OR 'transsexuality'/exp OR transsexualism:ab,ti OR transwoman:ab,ti OR 'trans women':ab,ti OR transwomen:ab,ti OR inequit*:ab,ti OR 'Insurance'/exp OR insuranc*:ab,ti OR 'socioeconomic index*':ab,ti OR 'marriage'/exp OR 'marital status':ab,ti OR gender*:ab,ti) AND ('neurosurgery'/exp OR neurosurg*:ab,ti OR 'spinal cord tumor'/exp OR 'spine tumor'/exp OR 'nervous system tumor'/exp OR ((spinal:ab,ti OR spine:ab,ti OR nervous:ab,ti OR nerve:ab,ti) AND (neoplasm*:ab,ti OR tumor*:ab,ti OR tumour*:ab,ti OR surg*:ab,ti))) AND

('retrospective study'/exp OR (retrospective NEAR/2 stud*) OR 'RCT':ab,ti OR (random* NEAR/2 control* NEAR/2 trial*) OR 'randomization'/exp OR (random* NEAR/2 allocat*) OR 'randomisation':ab,ti OR 'randomization':ab,ti OR 'cohort analysis'/exp OR (cohort NEAR/2 (analys* OR stud*)) OR (outcom* NEAR/2 analys*) OR 'case control study'/exp OR (case NEAR/2 (control OR stud* or report* or series))) AND ([1990-2020]/py)

Web of Science (Clarivate Analytics)

\#1 $\quad$ TS=("ethnic dispari*" OR "health disparit*" OR "health care disparit*" OR "healthcare disparit*" OR "health-care disparit*" OR "health status disparit*" OR "disparit* in health" OR "culturally competent care" OR "transcultural care" OR "delivery of health care" OR "disparit*" OR "health related quality of life" OR "health-related quality of life" OR "health services accessib*" OR "health service* need*" OR "health service* demand*" OR "patient acceptance" OR "patient attitude" OR "patient select*" OR "quality of health care" OR "social class*" OR "social norm*" OR "social determinants of health" OR "social disparit*" OR "social factor*" OR "social inequit*" OR "socioeconomic factor*" OR "socioeconomic* disadvantag*" OR "zip code" OR educat* OR "health literac*" OR "economic stabilit*" OR "household income" OR "food supply" OR "food insecur*") 


\begin{tabular}{|c|c|}
\hline \#2 & $\begin{array}{l}\text { TS }=(\text { health NEAR disparit*) OR TS=(health NEAR accessib*) OR } \\
\text { TS }=(\text { health* NEAR indigenous) }\end{array}$ \\
\hline \#3 & \#1 OR \#2 \\
\hline \#4 & 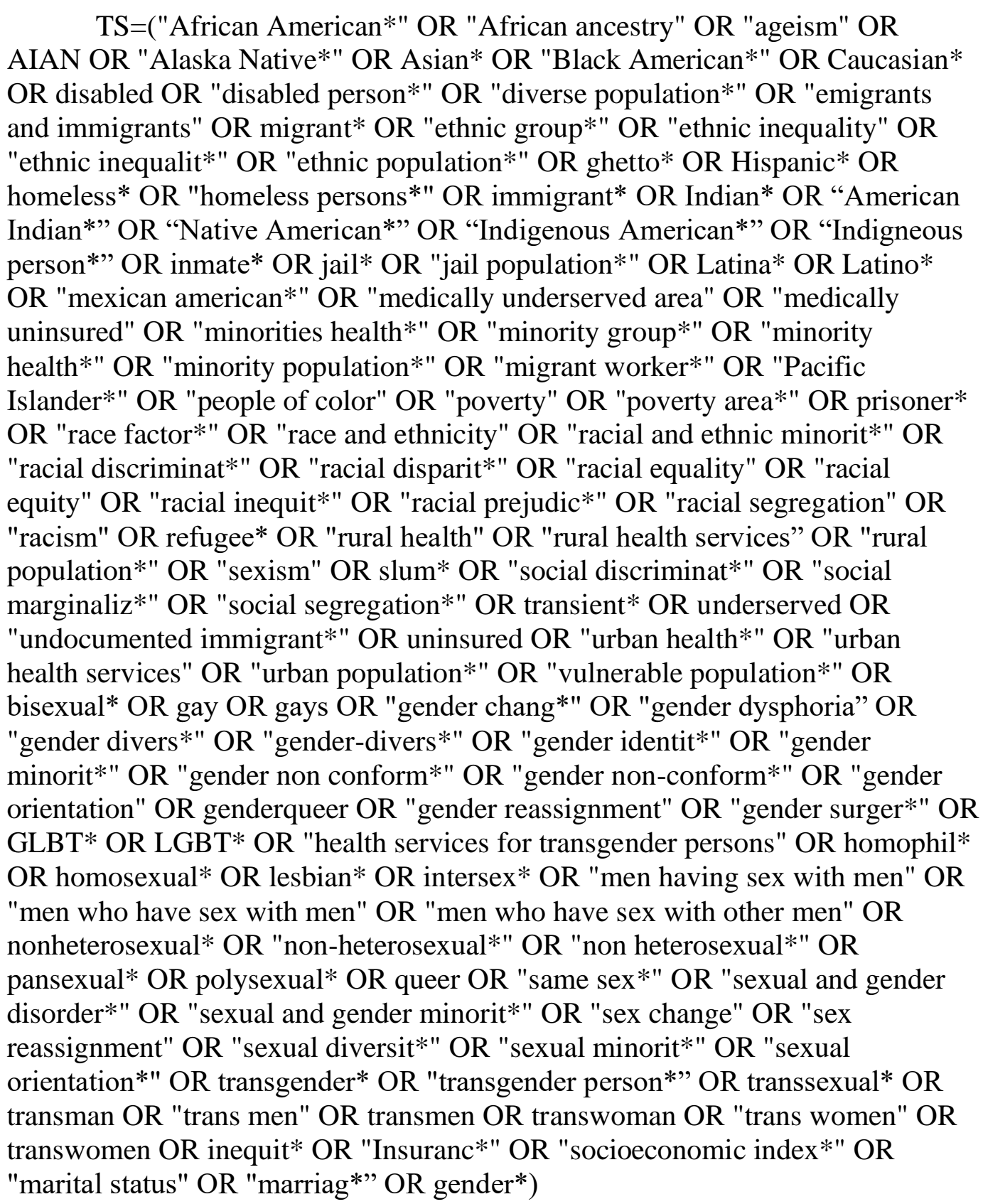 \\
\hline \#5 & $\begin{array}{l}\text { TS }=(\text { health servic* NEAR disabilit*) OR TS }=(\text { health servic* NEAR } \\
\text { disabl*) OR TS=(health servic* NEAR trangender*) OR TS=(health* NEAR } \\
\text { transgender*) }\end{array}$ \\
\hline
\end{tabular}




\begin{tabular}{|c|c|}
\hline \#6 & \#4 OR \#5 \\
\hline \#7 & $\begin{array}{l}\mathrm{TS}=(\text { neurosurg* OR }((\text { spinal OR spine OR nervous OR nerve*) AND } \\
(\text { neoplasm* OR tumor* OR tumour* OR surg*))) }\end{array}$ \\
\hline \#8 & $\begin{array}{l}\text { TS=("retrospective stud*” OR (retrospective AND stud*) OR } \\
\text { "Randomized Controlled Trial" OR "RCT" OR (random* AND control* AND } \\
\text { trial*) OR "Random Allocation" OR (random* and allocat*) OR "cohort stud*" } \\
\text { OR (cohort AND stud*) OR (outcom* AND analys*) OR “case control stud*” } \\
\text { OR "case-control stud*” OR (case AND control AND stud*) OR "Case Reports" } \\
\text { OR "case report*” OR (case AND report*) OR "case series" OR (case AND } \\
\text { series)) }\end{array}$ \\
\hline \#9 & \#3 AND \#6 AND \#7 AND \#8 AND Timespan: 1990-2020 \\
\hline
\end{tabular}

Cochrane CENTRAL (Wiley)

\begin{tabular}{|c|l|}
\hline$\# 1$ & \multicolumn{1}{|c|}{ ("ethnic dispari*":ti,ab,kw OR "health disparit*":ti,ab,kw OR "health care } \\
disparit*":ti,ab,kw OR "healthcare disparit*":ti,ab,kw OR "health-care \\
disparit*":ti,ab,kw OR "health status disparit*":ti,ab,kw OR "disparit* in \\
health":ti,ab,kw OR "culturally competent care":ti,ab,kw OR "transultural \\
care":ti,ab,kw OR "delivery of health care":ti,ab,kw OR "disparit*":ti,ab,kw OR \\
"health related quality of life":ti,ab,kw OR "health-related quality of life":ti,ab,kw \\
OR "health services accessib*":ti,ab,kw OR "health services needs and \\
demand":ti,ab,kw OR "patient acceptance":ti,ab,kw OR "patient attitude":ti,ab,kw \\
OR "patient select*":ti,ab,kw OR "quality of health care":ti,ab,kw OR "social \\
class*":ti,ab,kw OR "social norm*":ti,ab,kw OR "social determinants of \\
health":ti,ab,kw OR "social disparit*":ti,ab,kw OR "social factor*":ti,ab,kw OR \\
"social inequit*":ti,ab,kw OR "socioeconomic factor*":ti,ab,kw OR \\
"socioeconomically disadvantaged":ti,ab,kw OR "zip code":ti,ab,kw OR \\
educat*:ti,ab,kw OR "education level":ti,ab,kw OR "health literacy":ti,ab,kw OR \\
"economic stability":ti,ab,kw OR "household income":ti,ab,kw OR "food \\
supply":ti,ab,kw OR "food insecur*":ti,ab,kw)
\end{tabular}




\begin{tabular}{|c|c|}
\hline & 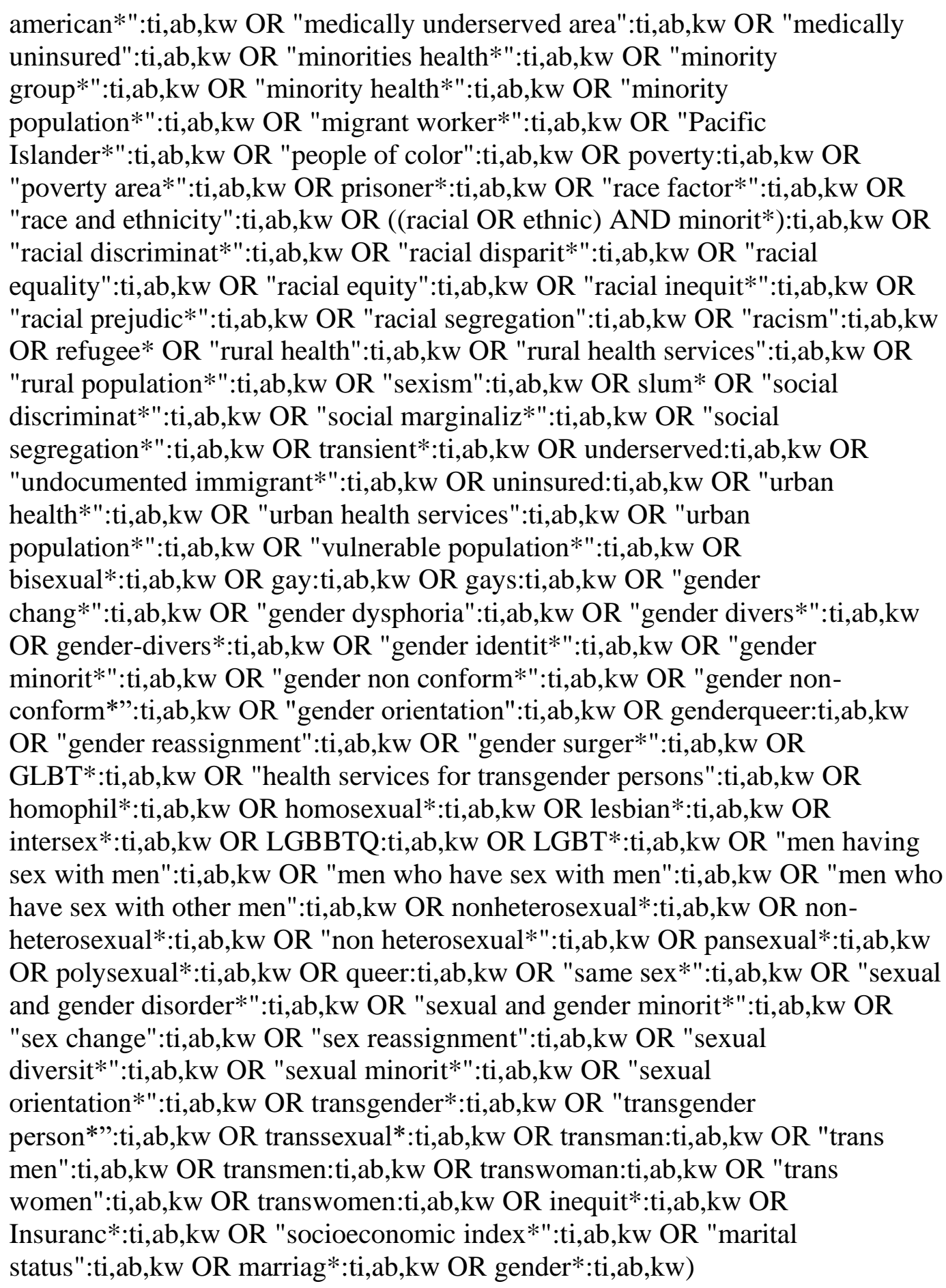 \\
\hline \#3 & $\begin{array}{l}\text { (neurosurg*:ti,ab,kw OR ((spinal:ti,ab,kw OR spine:ti,ab,kw OR } \\
\text { nervous:ti,ab,kw OR nerve*:ti,ab,kw) AND (neoplasm*:ti,ab,kw OR } \\
\text { tumor*:ti,ab,kw OR tumour*:ti,ab,kw OR surg*:ti,ab,kw))) }\end{array}$ \\
\hline \#4 & \#1 AND \#2 AND \#3 with Publication Year from 1990 to 2020, in Trials \\
\hline
\end{tabular}


CINAHL (EBSCOHost)

\begin{tabular}{|c|c|}
\hline \#1 & $\begin{array}{l}\text { TI ("ethnic disparit*" OR "health disparit*" OR "healthcare disparit*" OR } \\
\text { "health care disparit*" OR "health-care disparit*" OR "health status disparit*" } \\
\text { OR "disparit* in health" OR "culturally competent care" OR "delivery of health } \\
\text { care" OR disparit* OR "health related quality of life" OR "health-related quality } \\
\text { of life" OR "health services accessib*" OR "indigenous health*" OR "health } \\
\text { services needs and demand" OR "patient acceptance of health care" OR "patient } \\
\text { selection" OR "quality of health care" OR "social class*" OR "social norm*" OR } \\
\text { "social determinants of health" OR "social disparity*" OR "social factor*" OR } \\
\text { "social inequit*" OR "socioeconomic factor*" OR "socioeconomically } \\
\text { disadvantaged" OR "zip code" OR educat* OR "education level" OR "health } \\
\text { literacy" OR "economic stability" OR "household income" OR "food supply" OR } \\
\text { "food insecur*") }\end{array}$ \\
\hline \#2 & $\begin{array}{l}\text { AB ("ethnic disparit*" OR "health disparit*" OR "healthcare disparit*" } \\
\text { OR "health care disparit*" OR "health-care disparit*" OR "health status } \\
\text { disparit*" OR "disparit* in health" OR "culturally competent care" OR "delivery } \\
\text { of health care" OR disparit* OR "health related quality of life" OR "health-related } \\
\text { quality of life" OR "health services accessib*" OR "indigenous health*" OR } \\
\text { "health services needs and demand" OR "patient acceptance of health care" OR } \\
\text { "patient selection" OR "quality of health care" OR "social class*" OR "social } \\
\text { norm*" OR "social determinants of health" OR "social disparity*" OR "social } \\
\text { factor*" OR "social inequit*" OR "socioeconomic factor*" OR } \\
\text { "socioeconomically disadvantaged" OR "zip code" OR educat* OR "education } \\
\text { level" OR "health literacy" OR "economic stability" OR "household income" OR } \\
\text { "food supply" OR "food insecur*") }\end{array}$ \\
\hline \#3 & $\begin{array}{l}\text { (MH "healthcare disparities") OR (MH "Health status disparities") OR } \\
\text { (MH "Cultural Competence") OR (MM "Health Care Delivery") OR (MH } \\
\text { "Health Services Needs and Demand") OR (MH "Health Services Accessibility") } \\
\text { OR (MH "Health Services, Indigenous") OR (MH "Patient Selection") OR (MM } \\
\text { "Quality of Health Care") OR (MH "Social Class") OR (MH "Social } \\
\text { Determinants of Health") OR (MH "Socioeconomic Factors") OR (MH } \\
\text { "Economic Status") OR (MH "Education") OR (MH "Health Literacy") OR (MH } \\
\text { "Food Assistance") OR (MH "Food Security") }\end{array}$ \\
\hline \#4 & S1 OR S2 OR S3 \\
\hline \#5 & $\begin{array}{l}\text { TI ("African American*" OR "African ancestry" OR "ageism" OR AIAN } \\
\text { OR "Alaska Native*" OR Asian* OR "Black American*" OR Caucasian* OR } \\
\text { disabl* OR disabil* OR "disabled person*" OR "diverse population*" OR } \\
\text { emigrant* OR immigrant* OR refugee* OR "ethnic group*" OR "ethnic } \\
\text { inequalit*" OR "ethnic population*" OR ghetto* OR "health services for persons }\end{array}$ \\
\hline
\end{tabular}




\begin{tabular}{|c|c|}
\hline & $\begin{array}{l}\text { with disabilities" OR Hispanic* OR "hispanic american*" OR homeless* OR } \\
\text { "homeless person*" OR Indian* OR "indians, north american" OR "native } \\
\text { american*" or "north american indian*” OR "indigenous person*” OR } \\
\text { "indigenous people*" OR inmate* OR jail* OR "jail population*" OR Latina* } \\
\text { OR Latino* OR "mexican american*" OR "medically underserved area" OR } \\
\text { "medically uninsured" OR "minorities health*" OR "minority group*" OR } \\
\text { "minority health*" OR "minority population*" OR "migrant worker*" OR } \\
\text { "Pacific Islander*" OR "people of color" OR poverty OR "poverty area*” OR } \\
\text { prisoner* OR "race factor*" OR (race AND ethnicit*) OR ((racial OR ethnic) } \\
\text { AND minorit*) OR "racial discriminat*" OR "racial disparit*" OR "racial } \\
\text { equality" OR "racial equit*" OR "racial inequit*" OR "racial prejudic*" OR } \\
\text { "racial segregat*" OR "racism" OR "rural health*" OR "rural health services" OR } \\
\text { "rural population*" OR "sexism" OR slum* OR "social discriminat*" OR "social } \\
\text { marginaliz*" OR "social segregat*" OR underserved OR "undocumented } \\
\text { immigrant*" OR uninsured OR "urban health*" OR "urban health services" OR } \\
\text { "urban population*" OR "vulnerable population*" OR bisexual* OR gay OR gays } \\
\text { OR "gender change" OR "gender dysphoria" OR "gender divers*" OR "gender } \\
\text { divers*" OR "gender-divers*" OR "gender identit*" OR "gender minorit*" OR } \\
\text { "gender non conform*" OR "gender non-conform*" OR "gender orientation*" } \\
\text { OR genderqueer OR "gender reassignment" OR "gender surger*" OR GLBT* OR } \\
\text { LGBT* OR "health services for transgender person*" OR homophil* OR } \\
\text { homosexual* OR intersex* OR lesbian* OR "men having sex with men" OR } \\
\text { "men who have sex with men" OR "men who have sex with other men" OR } \\
\text { nonheterosexual* OR "non-heterosexual*" OR "non heterosexual*" OR } \\
\text { pansexual* OR polysexual* OR queer OR "same sex" OR "sexual and gender } \\
\text { disorder*" OR "sexual and gender minorit*" OR "sex change" OR "sex } \\
\text { reassignment" OR "sexual divers*” OR "sexual minorit*" OR "sexual } \\
\text { orientation*" OR transgender* OR "transgender person*" OR transsexual* OR } \\
\text { transman OR "trans men" OR transmen OR transwoman OR "trans women" OR } \\
\text { transwomen OR inequit* OR insuranc* OR "socioeconomic index*" OR "marital } \\
\text { status" OR gender*) }\end{array}$ \\
\hline \#6 & $\begin{array}{l}\text { AB ("African American*" OR "African ancestry" OR "ageism" OR AIAN } \\
\text { OR "Alaska Native*" OR Asian* OR "Black American*" OR Caucasian* OR } \\
\text { disabl* OR disabil* OR "disabled person*" OR "diverse population*" OR } \\
\text { emigrant* OR immigrant* OR refugee* OR "ethnic group*" OR "ethnic } \\
\text { inequalit*" OR "ethnic population*" OR ghetto* OR "health services for persons } \\
\text { with disabilities" OR Hispanic* OR "hispanic american*" OR homeless* OR } \\
\text { "homeless person*" OR Indian* OR "indians, north american" OR "native } \\
\text { american*” or "north american indian*” OR "indigenous person*” OR } \\
\text { "indigenous people*” OR inmate* OR jail* OR "jail population*" OR Latina* } \\
\text { OR Latino* OR "mexican american*" OR "medically underserved area" OR } \\
\text { "medically uninsured" OR "minorities health*" OR "minority group*" OR } \\
\text { "minority health*" OR "minority population*" OR "migrant worker*" OR } \\
\text { "Pacific Islander*" OR "people of color" OR poverty OR "poverty area*” OR }\end{array}$ \\
\hline
\end{tabular}




\begin{tabular}{|c|c|}
\hline & $\begin{array}{l}\text { prisoner* OR "race factor*" OR (race AND ethnicit*) OR ((racial OR ethnic) } \\
\text { AND minorit*) OR "racial discriminat*" OR "racial disparit*" OR "racial } \\
\text { equality" OR "racial equit*" OR "racial inequit*" OR "racial prejudic*" OR } \\
\text { "racial segregat*" OR "racism" OR "rural health*" OR "rural health services" OR } \\
\text { "rural population*" OR "sexism" OR slum* OR "social discriminat*" OR "social } \\
\text { marginaliz*" OR "social segregat*" OR underserved OR "undocumented } \\
\text { immigrant*" OR uninsured OR "urban health*" OR "urban health services" OR } \\
\text { "urban population*" OR "vulnerable population*" OR bisexual* OR gay OR gays } \\
\text { OR "gender change" OR "gender dysphoria" OR "gender divers*" OR "gender } \\
\text { divers*" OR "gender-divers*" OR "gender identit*" OR "gender minorit*" OR } \\
\text { "gender non conform*" OR "gender non-conform*" OR "gender orientation*" } \\
\text { OR genderqueer OR "gender reassignment" OR "gender surger*" OR GLBT* OR } \\
\text { LGBT* OR "health services for transgender person*" OR homophil* OR } \\
\text { homosexual* OR intersex* OR lesbian* OR "men having sex with men" OR } \\
\text { "men who have sex with men" OR "men who have sex with other men" OR } \\
\text { nonheterosexual* OR "non-heterosexual*" OR "non heterosexual*" OR } \\
\text { pansexual* OR polysexual* OR queer OR "same sex" OR "sexual and gender } \\
\text { disorder* OR "sexual and gender minorit*" OR "sex change" OR "sex } \\
\text { reassignment" OR "sexual divers*" OR "sexual minorit*" OR "sexual } \\
\text { orientation*" OR transgender* OR "transgender person*" OR transsexual* OR } \\
\text { transman OR "trans men" OR transmen OR transwoman OR "trans women" OR } \\
\text { transwomen OR inequit* OR insuranc* OR "socioeconomic index*" OR "marital } \\
\text { status" OR gender*) }\end{array}$ \\
\hline \#7 & $\begin{array}{l}\text { (MH "Blacks") OR (MH "Ageism") OR (MH "Asians") OR (MH "Native } \\
\text { Americans") OR (MH "Indigenous Peoples") OR (MH "Health Services, } \\
\text { Indigenous") OR (MH "Indigenous Health") OR (MH "Whites") OR (MH } \\
\text { "Health Services for Persons with Disabilities") OR (MH "LGBTQ Persons") OR } \\
\text { (MH "Transgender Persons") OR (MH "Disabled") OR (MH "Rural Population") } \\
\text { OR (MH "Immigrants, Illegal") OR (MH "Immigrants") OR (MH "Emigration } \\
\text { and Immigration") OR (MH "Ethnic Groups") OR (MH "Poverty Areas") OR } \\
\text { (MH "Hispanics") OR (MH "Homeless Persons") OR (MH "Homelessness") OR } \\
\text { (MH "Prisoners") OR (MH "Correctional Health Services") OR (MH } \\
\text { "Correctional Facilities") OR (MH "Medically Underserved Area") OR (MH } \\
\text { "Medically Uninsured") OR (MH "Minority Groups") OR (MH "Sexual and } \\
\text { Gender Minorities") OR (MH "Transients and Migrants") OR (MH "Race } \\
\text { Factors") OR (MH "Racism") OR (MH "Refugees") OR (MH "Rural Health") } \\
\text { OR (MH "Rural Health Services") OR (MH "Sexism") OR (MH } \\
\text { "Discrimination") OR (MH "Medically Underserved") OR (MH "Urban } \\
\text { Population") OR (MH "Urban Health Services") OR (MH "Urban Health") OR } \\
\text { (MH "Hospitals, Urban") OR (MH "Gay Persons") OR (MH "Gay Men") OR } \\
\text { (MH "Men Who Have Sex With Men") OR (MH "Bisexuals") OR (MH } \\
\text { "Bisexuality") OR (MH "Gender Affirmation Procedures") OR (MH "Sexual and } \\
\text { Gender Disorders") OR (MH "Gender Affirmation Surgery") OR (MH "Gender } \\
\text { Identity") OR (MH "Gender Dysphoria") OR (MH "Gender Nonconformity") OR }\end{array}$ \\
\hline
\end{tabular}




\begin{tabular}{|c|c|}
\hline & $\begin{array}{l}\text { (MH "Sexuality") OR (MH "Sexual Identity") OR (MH "Intersex Persons") OR } \\
\text { (MH "Lesbians") OR (MH "Transsexuals") OR (MH "Transsexualism") OR (MH } \\
\text { "Insurance") OR (MH "Insurance, Health") OR (MH "Marital Status") }\end{array}$ \\
\hline \#8 & S5 OR S6 OR S7 \\
\hline$\# 9$ & $\begin{array}{l}\text { (TI (neurosurg* OR ((spinal OR spine OR nervous OR nerve*) AND } \\
\text { (neoplasm* OR tumor* OR tumour* OR surg*)))) OR (AB (neurosurg* OR } \\
\text { ((spinal OR spine OR nervous OR nerve*) AND (neoplasm* OR tumor* OR } \\
\text { tumour* OR surg*))) OR ((MH "Neurosurgery+") OR (MM "Spinal Cord } \\
\text { Neoplasms") OR (MM "Spinal Neoplasms") OR (MH "Spinal Cord Injuries+") } \\
\text { OR (MH "Nervous System Neoplasms+")) }\end{array}$ \\
\hline$\# 1$ & $\begin{array}{l}\text { TI ("retrospective stud*" OR (retrospective AND stud*) OR } \\
\text { "Randomized Controlled Trial" OR "RCT" OR (random* AND control* AND } \\
\text { trial*) OR "Random Allocat*" OR (random* and allocat*) OR "cohort stud*" OR } \\
\text { (cohort AND stud*) OR (outcom* AND analys*) OR "case control stud*" OR } \\
\text { (case AND control AND stud*) OR "case report*" OR (case AND report*) OR } \\
\text { "case series" OR (case AND series)) }\end{array}$ \\
\hline$\# 1$ & $\begin{array}{l}\text { AB ("retrospective stud*" OR (retrospective AND stud*) OR } \\
\text { "Randomized Controlled Trial" OR "RCT" OR (random* AND control* AND } \\
\text { trial*) OR "Random Allocat*" OR (random* and allocat*) OR "cohort stud*" OR } \\
\text { (cohort AND stud*) OR (outcom* AND analys*) OR "case control stud*" OR } \\
\text { (case AND control AND stud*) OR "case report*" OR (case AND report*) OR } \\
\text { "case series" OR (case AND series)) }\end{array}$ \\
\hline$\# 1$ & $\begin{array}{l}\text { (MH "Retrospective Design") OR (MH "Retrospective Panel Studies") } \\
\text { OR (MH "Randomized Controlled Trials") OR (MH "Random Assignment") OR } \\
\text { (MH "Prospective Studies") OR (MH "Outcome Assessment") OR (MH "Case } \\
\text { Control Studies") OR (MH "Case Studies") }\end{array}$ \\
\hline$\# 1$ & S10 OR S11 OR S12 \\
\hline$\# 1$ & $\begin{array}{l}\text { S4 AND S8 AND S9 AND S13, Published Date: 19900101-20201231; } \\
\text { English Language }\end{array}$ \\
\hline
\end{tabular}

Clinicaltrials.gov

disparity AND (racial OR gender OR minority) | Nervous System Diseases Primary completion from $01 / 01 / 1990$ to $12 / 03 / 2020$

National Institute for Health and Care Excellence (NICE) disparity AND (racial OR gender OR minority) AND neurosurg* 
Publication date 1/1/1990-12/3/2020 